\title{
Constellation Randomization Achieves Transmit Diversity for Single-RF Spatial Modulation
}

\author{
Christos Masouros, Senior Member, IEEE, and Lajos Hanzo, Fellow, IEEE
}

\begin{abstract}
4 Abstract-The performance of spatial modulation (SM) is known 5 to be dominated by the minimum Euclidean distance (MED) in the 6 received SM constellation. In this paper, a symbol-scaling tech7 nique is proposed for $\mathbf{S M}$ in the multiple-input-multiple-output 8 (MIMO) channel that enhances the MED to improve the perfor9 mance of SM. This is achieved by forming fixed sets of candidate 10 prescaling factors for the transmit antennas (TAs), which are ran11 domly generated and are known at both the transmitter and the re12 ceiver. For a given channel realization, the transmitter chooses the 13 specific set of factors that maximizes the MED. Given the channel 14 state information (CSI) readily available at the receiver for detec15 tion, the receiver independently chooses the same set of prescaling 16 factors and uses them for the detection of both the antenna index 17 (AI) and the symbol of interest. We analytically calculate the 18 attainable gains of the proposed technique, in terms of its transmit 19 diversity order, based on both the distribution of the MED and on 20 the theory of classical order statistics. Furthermore, we show that 21 the proposed scheme offers a scalable performance-complexity 22 tradeoff for SM by varying the number of candidate sets of pre23 scaling factors, with significant performance improvements, com24 pared to conventional SM.
\end{abstract}

25 Index Terms-Constellation shaping, multiple-input single26 output, prescaling, spatial modulation (SM).

\section{INTRODUCTION}

28

${ }_{30} \mathbf{T}$ RADITIONAL spatial multiplexing has been shown to improve the capacity of the wireless channel by exploiting 31 multiantenna transmitters [1]. More recently, spatial modula32 tion (SM) has been explored as a means of implicitly encoding 33 information in the index of the specific antenna activated for 34 the transmission of the modulated symbols, offering a low35 complexity alternative [2]. Its central benefits include the ab36 sence of interantenna interference (IAI) and the fact that it only 37 requires a subset (down to one) of radio-frequency (RF) chains 38 compared to spatial multiplexing. Accordingly, the interantenna 39 synchronization is also relaxed. Early work has focused on the 40 design of receiver algorithms for minimizing the bit error rate 41 (BER) of SM at a low complexity [2]-[6]. Matched filtering

Manuscript received September 30, 2014; revised June 3, 2015 and November 10, 2015; accepted December 27, 2015. This work was supported by the Royal Academy of Engineering, U.K. The review of this paper was coordinated by Prof. Y. Gong.

C. Masouros is with the Department of Electrical and Electronic Engineering, University College London, London WC1E 7JE, U.K. (e-mail: c.masouros@ ucl.ac.uk)

L. Hanzo is with the School of Electronics and Computer Science, University of Southampton, Southampton SO17 1BJ, U.K. (e-mail: 1h@ecs.soton.ac.uk).

Color versions of one or more of the figures in this paper are available online at http://ieeexplore.ieee.org.

Digital Object Identifier 10.1109/TVT.2015.2513380 is shown to be a low-complexity technique for detecting the 42 antenna index (AI) used for SM [2]. A maximum-likelihood 43 (ML) detector is introduced in [4] for reducing the complexity 44 of classic spatial multiplexing ML detectors. Compressive sens- 45 ing and reduced-space sphere detection have been discussed for 46 SM in [5] and [6] for further complexity reduction.

In addition to receive processing, recent work has also pro- 48 posed constellation shaping for SM [7]-[15]. Specifically, in 49 [7], the transmit diversity of coded SM is analyzed for differ- 50 ent spatial constellations, which represent the legitimate sets 51 of activated transmit antennas (TAs). Furthermore, Yang [8] 52 discusses symbol constellation optimization for minimizing the 53 BER. Indeed, spatial- and symbol-constellation shaping are 54 discussed separately, as aforementioned. By contrast, the design 55 of the received SM constellation that combines the choice of 56 the TA, as well as the transmit symbol constellation, is the 57 focus of this paper. Precoding-aided approaches that combine 58 SM with spatial multiplexing are studied in [11] and [12]. A 59 number of constellation-shaping schemes [9]-[15] have also 60 been proposed for the special case of SM, which is referred to 61 as space shift keying, where the information is only carried in 62 the spatial domain, by the activated AI. Their application to the 63 SM transmission, where the transmit waveform is modulated, 64 is nontrivial.

Closely related work has focused on shaping the receive SM 66 constellation by means of symbol prescaling at the transmitter, 67 aiming at maximizing the minimum Euclidean distance (MED) 68 in the received SM constellation [17]-[19]. The constellation- 69 shaping approach in [17] and [18] aims at fitting the receive 70 SM constellation to one of the existing optimal constellation 71 formats in terms of minimum distance, such as, e.g., quadrature 72 amplitude modulation (QAM). Due to the strict constellation 73 fitting requirement imposed on both the amplitude and the 74 phase, this prescaling relies on the inversion of the channel 75 coefficients. In the case of ill-conditioned channels, this sub- 76 stantially increases the power associated to the transmit constel- 77 lation and therefore requires scaling factors for normalizing the 78 transmit power, which, however, reduces the received signal- 79 to-noise ratio (SNR). This problem has been alleviated in [19], 80 where a constellation-shaping scheme based on phase-only 81 scaling is proposed. Nevertheless, the constellation shaping 82 used in the aforementioned schemes is limited in the sense that 83 it only applies to multiple-input-single-output (MISO) systems 84 where a single symbol is received for each transmission, and 85 thus, the characterization and shaping of the receive SM con- 86 stellation is simple. The application of constellation shaping in 87 the multiple-input-multiple-output (MIMO) systems is still an 88 open problem. 


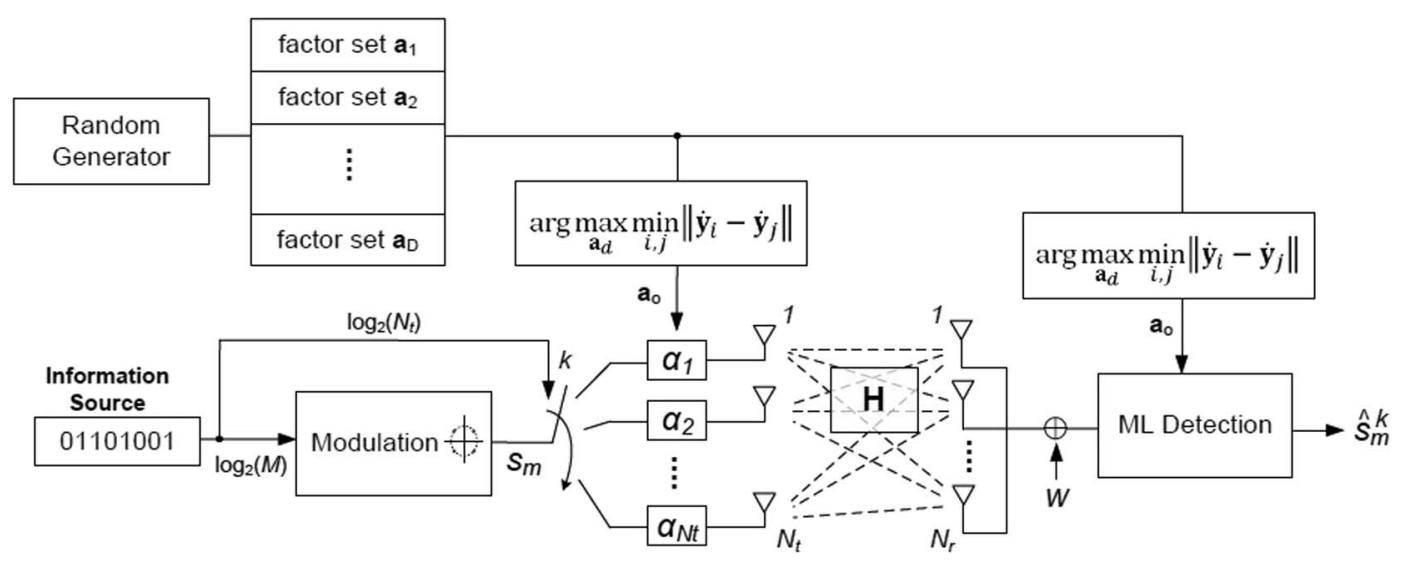

Fig. 1. Block diagram of SM transceiver with constellation randomization (SM-CR).

90 In line with the aforementioned challenges, in this paper, we 91 introduce a new transmit prescaling (TPS) scheme, where the 92 received constellation fitting problem is relaxed. As opposed 93 to the aforementioned strict constellation fitting approaches, 94 here, the received SM constellation is randomized by TPS for 95 maximizing the MED between its points for a given channel. 96 In more detail, a number of randomly generated candidate sets 97 of TPS factors are formed offline, which are known to both the 98 transmitter and the receiver. Each of these sets is normalized, 99 so that the average transmit power remains unchanged, and 100 yields a different receive constellation for a certain channel 101 realization. For a given channel, the transmitter then selects that 102 particular set of TPS factors that yields the SM constellation 103 having the maximum MED. By doing so, the TPS alleviates 104 the cases where different TAs yield similar received symbols 105 and thus improves the reliability of symbol detection. At the 106 receiver, by exploiting the channel state information (CSI) 107 readily available for detection, the detector selects the same set 108 of TPS factors to form the received constellation and applies 109 an ML test to estimate the data. The explicit benefit of the 110 aforementioned methodology is that it extends the idea of re111 ceive SM constellation shaping to the MIMO scenarios having 112 multiple antennas at the receiver, and it will be shown that it 113 introduces additional transmit diversity gains and improves the 114 power efficiency of the SM system. Against this background, $11 \bar{\S}$ we list the main contributions of this paper as follows.
- We propose a new per-antenna TPS scheme for SM-aided point-to-point MIMO transmission that improves the attainable performance.

- We analytically derive a tight upper bound of the transmit diversity gains obtained by the proposed technique, based on the distribution of the MED in the received constellation for transmission over a frequency-flat Rayleigh distributed channel.

- We analyze the computational complexity of the proposed scheme to demonstrate how a scalable performancecomplexity tradeoff can be provided by the proposed technique, when adapting the number of candidate sets of TPS factors.

- Using the aforementioned performance and complexity analyses, we study the power efficiency of the proposed scheme in comparison to conventional SM. We introduce a power efficiency metric that combines the transmit 133 power, the achieved throughput, and the computational 134 complexity imposed to quantify the improved power ef- 135 ficiency offered by the proposed scheme.

The remainder of this paper is organized as follows. Section II 137 presents the MIMO system model and introduces the SM trans- 138 mission. Section III details the proposed TPS scheme, while 139 in Section IV, we present our analytical study of the obtained 140 transmit diversity gains of the proposed scheme. Sections V 141 and VI detail the complexity calculation and the study of the 142 attainable power efficiency. Section VII presents our numerical 143 results, and finally, our conclusions are offered in Section VIII. 144

\section{System Model and Spatial Modulation}

\section{A. System Model}

Consider a MIMO system where the transmitter and receiver 147 are equipped with $N_{t}$ and $N_{r}$ antennas, respectively. For sim- 148 plicity, unless stated otherwise, in this paper, we assume that 149 the transmit power budget is limited to unity, i.e., $P=1$. See 150 [20]-[22] for extensive reviews and tutorials on the basics and 151 state-of-the-art on SM. Here, we focus on the single-RF-chain 152 SM approach, where the transmit vector is in the all-but-one 153 zero form $\mathbf{s}_{m}^{k}=\left[0, \ldots, s_{m}, \ldots, 0\right]^{T}$, where the notation [.] $]^{T} 154$ denotes the transpose operator. Here, $s_{m}, m \in\{1, \ldots, M\}$ is 155 a symbol taken from an $M$-order modulation alphabet that 156 represents the transmitted waveform in the baseband domain 157 conveying $\log _{2}(M)$ bits, and $k$ represents the index of the ac- 158 tivated TA (the index of the nonzero element in $\mathbf{s}_{m}^{k}$ ) conveying 159 $\log _{2}\left(N_{t}\right)$ bits in the spatial domain. Clearly, since $\mathbf{s}$ is an all- 160 zero vector apart from $s_{m}^{k}$, there is no IAI.

The per-antenna TPS approach, which is the focus of this 162 paper, is shown in Fig. 1. The signal fed to each TA is scaled by 163 a complex-valued coefficient $\alpha_{k}, k \in\left\{1, \ldots, N_{t}\right\}$, for which 164 we have $E\left\{\left|\alpha_{k}\right|\right\}=1$, where $|x|$ denotes the amplitude of a 165 complex number $x$, and $E\{$.$\} denotes the expectation operator. 166$ Defining the MIMO channel vector as $\mathbf{H}$, with elements $h_{i, j} 167$ representing the complex channel coefficient between the $i$ th 168 TA to the $j$ th receive antenna (RA), the received symbol vector 169 can be written as

$$
\mathbf{y}=\mathbf{H A} \mathbf{s}_{m}^{k}+\mathbf{w}
$$


171 where $\mathbf{w} \sim \mathcal{C N}\left(0, \sigma^{2} \mathbf{I}\right)$ is the additive white Gaussian noise 172 component at the receiver, with $\mathcal{C N}\left(\mu, \sigma^{2}\right)$ denoting the cir173 cularly symmetric complex Gaussian distribution with mean $\mu$ 174 and variance $\sigma^{2}$. Furthermore, $\mathbf{A}=\operatorname{diag}(\mathbf{a}) \in \mathbb{C}^{N_{t} \times N_{t}}$ is the 175 TPS matrix with $\mathbf{a}=\left[\alpha_{1}, \alpha_{2}, \ldots, \alpha_{N_{t}}\right]$, and $\operatorname{diag}(\mathbf{x})$ represents 176 the diagonal matrix with its diagonal elements taken from 177 vector X. Note that the diagonal structure of $\mathbf{A}$ guarantees 178 having a transmit vector $\mathbf{t}=\mathbf{A s}$ with a single nonzero element, 179 so that the single-RF-chain aspect of SM is preserved.

180 At the receiver, a joint ML detection of both the TA index 181 and the transmit symbol is obtained by the minimization

$$
\begin{aligned}
{\left[\hat{s}_{m}, \hat{k}\right] } & =\arg \min _{i}\left\|\mathbf{y}-\mathbf{y}_{i}\right\| \\
& =\arg \min _{m, k}\left\|\mathbf{y}-\mathbf{H A} \mathbf{s}_{m}^{k}\right\|
\end{aligned}
$$

182 where $\|\mathbf{x}\|$ denotes the norm of vector $\mathbf{x}$, and $\mathbf{y}_{i}$ is the $i$ th con183 stellation point in the received SM constellation. By exploiting 184 the specific structure of the transmit vector, this can be further 185 simplified to

$$
\left[\hat{s}_{m}, \hat{k}\right]=\arg \min _{m, k}\left\|\mathbf{y}-\mathbf{h}_{k} \alpha_{m}^{k} s_{m}\right\|
$$

186 where $\mathbf{h}_{k}$ denotes the $k$ th column of matrix $\mathbf{H}$, and $\alpha_{m}^{k}$ is the 187 TPS coefficient of the $k$ th TA. It is widely recognized that the 188 performance of the detection, as explained earlier, is dominated 189 by the MED between adjacent constellation points $\dot{\mathbf{y}}_{i}, \dot{\mathbf{y}}_{j}$ in the 190 receive $\mathrm{SM}$ constellation, i.e.,

$$
d_{\min }=\min _{i, j}\left\|\mathbf{y}_{i}-\mathbf{y}_{j}\right\|^{2}, i \neq j .
$$

191 Accordingly, to improve the likelihood of correct detection, 192 constellation-shaping TPS schemes for SM aim at maximizing 193 this MED. The optimum TPS matrix $\mathbf{A}^{*}$ can be found by 194 solving the optimization

$$
\begin{aligned}
& \mathbf{A}^{*}=\arg \max _{\mathbf{A}} \min _{i, j}\left\|\mathbf{y}_{i}-\mathbf{y}_{j}\right\|^{2}, i \neq j \\
& \text { s.t.c. } \quad \operatorname{trace}\left(\mathbf{A}^{* H} \mathbf{A}^{*}\right) \leq P
\end{aligned}
$$

195 and, additionally for single-RF-chain SM, subject to $\mathbf{A}^{*}$ having 196 a diagonal structure. As aforementioned, $\mathbf{A}^{H}$ and trace(A) 197 represent the Hermitian transpose and trace of matrix A, re198 spectively. The aforementioned optimization, however, is an 199 NP-hard problem, which makes finding the TPS factors pro200 hibitively complex and motivates the conception of lower com201 plexity suboptimal techniques.

\section{B. Prescaling for the MISO Channel}

203 In line with the aforementioned discussions, in [17], a 204 prescaling scheme is proposed for the MISO channel. Assum205 ing a channel vector $\mathbf{h}$, the receive SM constellation is fitted to 206 a $Q$-QAM constellation with $Q=N_{t} M$ by choosing

$$
\tilde{\alpha}_{m}^{k}=\frac{q_{(m-1) M+k}\|\mathbf{h}\|}{h_{k} s_{m} \sqrt{N_{t}}}
$$

207 where $q_{i}$ is the $i$ th constellation point in the $Q$-QAM constel208 lation, and the factor $\|\mathbf{h}\| / \sqrt{N_{t}}$ is used for normalizing the 209 receive constellation so that $E\{|q|\}=1$.
We note that, while the scaling in (6) normalizes the receive 210 constellation, it does not normalize the transmit power. There- 211 fore, power-normalized scaling coefficients should be used in 212 the form

$$
\alpha_{m}^{k}=\frac{\tilde{\alpha}_{m}^{k}}{\|\tilde{\mathbf{a}}\|} .
$$

Nevertheless, it can be seen that for ill-conditioned channel 214 coefficients, even for just one of the TAs, this leads to low 215 power-scaling factors $f=1 /\|\tilde{\mathbf{a}}\|$, which limits the obtainable 216 performance. Finally, note that $\alpha_{m}^{k}$ are data dependent for this 217 approach, as evidenced by the index $m$, which does not allow 218 for a fixed per-antenna scaling coefficient, as shown in Fig. 1. 219 Most importantly, the aforementioned strict constellation fitting 220 cannot be extended to systems having multiple RAs, since 221 the inversion of the full channel matrix $\mathbf{H}$ would result in 222 nonzero elements in the transmit vector $\mathbf{t}$, which means that 223 all TAs are used. Therefore, the important benefit of single-RF 224 transmission of SM is lost.

An alternative is shown in [19], again for the MISO channel, 226 where the scaling factors are in the form

$$
\begin{aligned}
\alpha_{k} & =e^{j \varphi_{k}} \\
\varphi_{k} & =\theta_{i}-\vartheta_{k}
\end{aligned}
$$

where $\vartheta_{k}$ is the phase of the $k$ th channel, and $\theta_{i}$ is the $i$ th angle 228 taken from an equally spaced angle arrangement within $[0,2 \pi) 229$ in the form

$$
\theta_{i}=\frac{2 \pi}{N_{t} M}(i-1), i \in\left\{1, \ldots, N_{t}\right\}
$$

In this way, the phases of the points in the receive SM con- 231 stellation become equispaced, hence maintaining a minimum 232 for the Euclidean distances in the constellation.

Aside from their individual limitations and the fact that they 234 are suboptimal, the aforementioned prescaling methods are lim- 235 ited by the fact that they apply solely to MISO systems relying 236 on a single RA and cannot be readily extended to the case of 237 MIMO SM transmission, hence lacking receive diversity. $\quad 238$

\section{Proposed Constellation Randomization PRESCALING (SM-CR)}

To alleviate the drawbacks of the aforementioned techniques, 241 we propose an adaptive TPS technique that randomizes the re- 242 ceived SM constellation. The proposed constellation randomiza- 243 tion (CR) simply selects the "best" from a number of randomly 244 generated sets of per-antenna TPS factors, with the aim of im- 245 proving the resulting MED. By allowing the randomization of 246 the amplitude and phase of the effective channel that combines 247 the TPS factor and the channel gains of the TA, the proposed 248 scheme relaxes the constellation optimization problem and 249 facilitates a better solution for the maximization of $d_{\min }$. In ad- 250 dition, through the aforementioned randomization and selection 251 of the appropriate TPS factors, the proposed scheme critically 252 improves the transmit diversity of the SM system, as will 253 be shown analytically in the following section. The proposed 254 scheme involves the steps as analyzed in the following. 


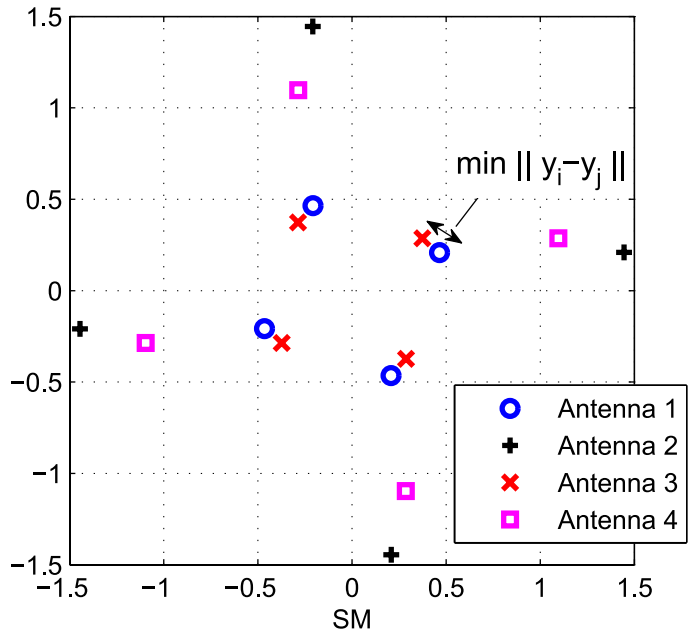

Fig. 2. Received constellation for a $4 \times 1$ MISO with SM and SM-CR for 4QAM.

\section{A. Formation of Candidate Prescaling Sets}

257 First, a number of $D$ candidate TPS vectors are generated 258 randomly in the form $\mathbf{a}_{d}$, where $d \in[1, D]$ denotes the index 259 of the candidate set, and $\mathbf{a}_{d}$ is formed by the elements $\alpha_{m}^{k(d)} \sim$ $260 \mathcal{C N}(0,1)$. These are made available to both the transmitter and 261 the receiver once, in an offline fashion before transmission. 262 These assist in randomizing the received constellation, which 263 is most useful in the cases where two points in the constellation 264 of $\mathbf{H s}_{m}^{k}, m \in[1, M], k \in\left[1, N_{t}\right]$ happen to be very close. To 265 ensure that the average transmit power remains unchanged, the 266 scaling factors are normalized as in (7). It is important to reit267 erate that, in this work, we focus on power-normalized scaling 268 factors, and hence, the proposed scheme does not constitute a 269 power-allocation scheme. This allows us to isolate the diversity 270 gains from the power and coding gains in our analysis in the 271 following section. In the generalized case, power allocation 272 could be applied on top of the prescaling, by employing a 273 diagonal power-allocation matrix, while the resulting diversity 274 gains would not change.

\section{B. Selection of Prescaling Vector}

276 For a given channel, based on the knowledge of vectors $\mathbf{a}_{d}$, 277 both the transmitter and the receiver can determine the received 278 SM constellation for every $d$ by calculating the set of $[m, k]$ 279 possibilities in

$$
\hat{\mathbf{y}}=\mathbf{H} \mathbf{A}_{d} \mathbf{s}_{m}^{k}
$$

280 where $\mathbf{A}_{d}=\operatorname{diag}\left(\mathbf{a}_{d}\right)$ is the diagonal matrix that corresponds 281 to the candidate set $\mathbf{a}_{d}$. Then, for the given channel coefficients, 282 the transmitter and receiver can independently choose the scal283 ing vector $\mathbf{a}_{o}$, for which

$$
\mathbf{a}_{o}=\arg \max _{d} \min _{\substack{m_{1}, m_{2}, k_{1}, k_{2} \\\left\{m_{1}, k_{1}\right\} \neq\left\{m_{2}, k_{2}\right\}}}\left\|\mathbf{H} \mathbf{A}_{d} \mathbf{s}_{m_{1}}^{k_{1}}-\mathbf{H} \mathbf{A}_{d} \mathbf{s}_{m_{2}}^{k_{2}}\right\|^{2} .
$$

284 The transmitter then sends $\mathbf{t}=\mathbf{A}_{o} \mathbf{s}_{m}^{k}$, with $\mathbf{A}_{o}=\operatorname{diag}\left(\mathbf{a}_{o}\right)$, 285 and the receiver applies the ML detector according to

$$
\left[\hat{s}_{m}, \hat{k}\right]=\arg \min _{m, k}\left\|\mathbf{y}-\mathbf{H} \mathbf{A}_{o} \mathbf{s}_{m}^{k}\right\|
$$

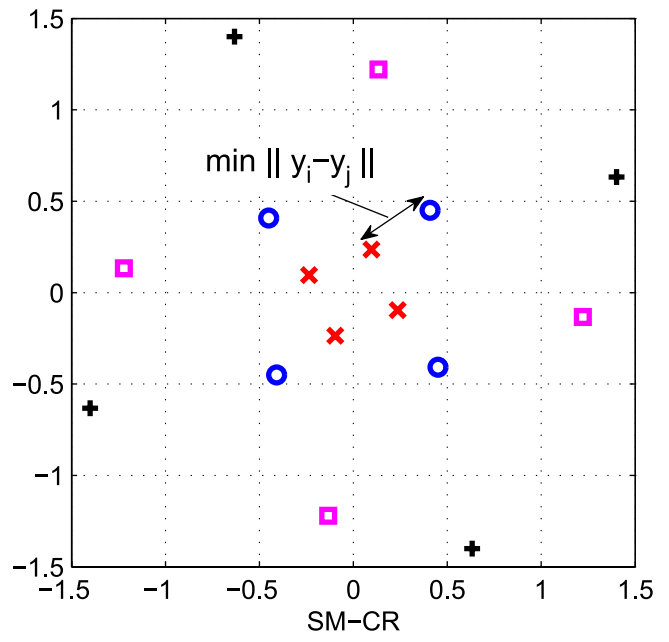

As mentioned earlier, since the channel coefficients are esti- 286 mated at the receiver for detection [2]-[6], (12) can be used to 287 derive the aforementioned factors independently at the receiver. 288 Therefore, no feed forwarding of $\alpha_{m}^{k(d)}$ or the index $d$ is 289 required. Indeed, for equal channel coefficients available at the 290 transmitter and receiver, they both select the same TPS vector 291 $\mathbf{a}_{o}$ independently, as per (12). Alternatively, to dispose of the 292 need for CSI at the transmitter (CSIT), the receiver can indeed 293 select the best scaling factors using (12) and feed the index of 294 the selected scaling vector $\mathbf{a}_{o}$ out of the $D$ candidates back 295 to the transmitter, using $\left\lceil\log _{2} D\right\rceil$ bits. In comparison to the 296 closely related works in [17]-[19], this provides the proposed 297 scheme with the advantage of a reduced transmit complexity 298 that, instead of CSIT acquisition and prescaling optimization, 299 involves the detection of $\left\lceil\log _{2} D\right\rceil$ bits at the end of every 300 channel coherence period, and a single complex multiplication 301 of the classically modulated symbol $s_{m}$ with the prescaling 302 factor $a_{m}^{k}$ in the form shown in (3).

303

The intuitive benefits of the proposed scheme in the MED of 304 the received SM constellation are shown in Fig. 2 for a $(4 \times 1)-305$ element MISO system employing 4QAM modulation at high 306 SNR, where the original receive SM constellation without TPS 307 is shown in the left-hand side, and the constellation after the 308 selection in (12) is illustrated on the right-hand side. A clear 309 increase in the MED can be observed, without increasing the 310 average transmit power. In fact, for the example in Fig. 2, a 311 slight reduction of the power in the symbols denoted by " $x$ " 312 can be observed, which, nevertheless, increases the MED in the 313 constellation.

Observe in Fig. 2 that while suboptimal in the constellation 315 design sense, the proposed TPS enhances the MED in the 316 constellation with respect to conventional SM, while imposing 317 a conveniently scalable complexity as per the size of candidate 318 sets $D$. It is evident that the gains in the MED for the proposed 319 scheme are dependent on the set size $D$ of the candidate 320 TSP vector sets $\mathbf{a}_{d}$ to choose from. An indicative result of 321 this dependence is shown in Fig. 3, where the average gains 322 in the MED are shown, with increasing numbers of $D$ for 323 different transmission scenarios. Theoretically derived upper 324 bounds for these gains for $N_{r}=1, N_{r}=2$, and $N_{r}=4$, based 325 


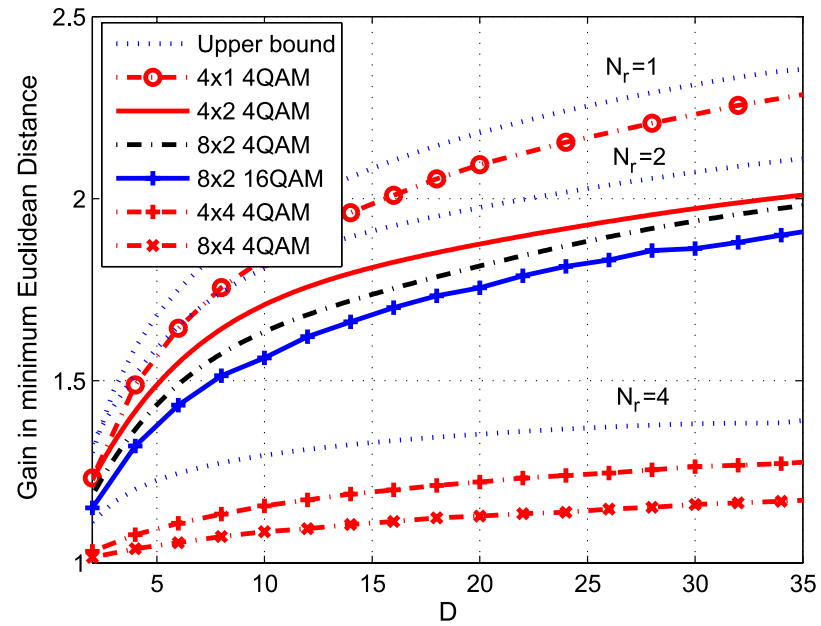

Fig. 3. Gain $G$ in average MED for SM-CR with respect to SM for increasing $D$.

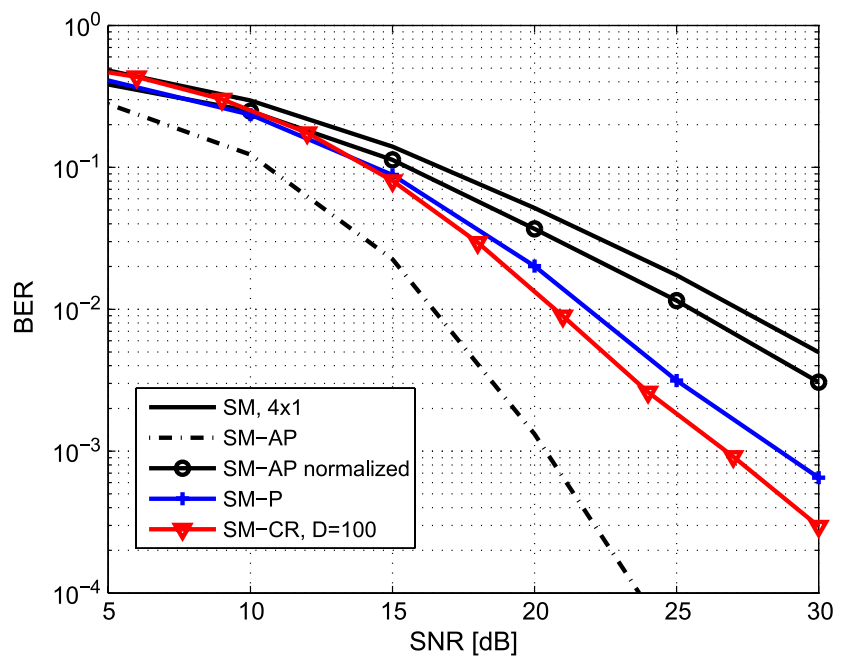

Fig. 4. BER versus SNR for a $(4 \times 1)$ MISO with SM, SM-AP [17], SM-P [19], and SM-CR with $D=100$ for 4QAM.

326 on Theorem 1 of the following section, are also shown in the 327 figure and will be detailed in the following. It can be seen that, 328 for low values of $D$, significant MED benefits are obtained by 329 increasing the number of candidates, while the gains saturate in 330 the region of higher values of $D$. This justifies the choice of low 331 values of $D$ to constrain the computational complexity involved 332 in the search in (12). In the results that follow, we explore the 333 error rates and complexity and their tradeoff in terms of power 334 efficiency as a means of optimizing the value of $D$ for different 335 performance targets.

\section{DIVERSITY ANALYSIS}

\section{A. Transmit Diversity}

338 The proposed CR scheme leads to an increase in the transmit 339 diversity gains. That is, while the transmit diversity of the 340 single-RF SM is known to be one [7], the proposed TPS 341 introduces an amplitude-phase diversity in the transmission, 342 due to the existence of $D$ candidate sets of TPS factors from 343 which to choose. The system is said to have a diversity order 344 of $\delta$, if the BER decays with $\gamma^{-\delta}$ in the high-SNR region, 345 with $\gamma$ being the SNR (see Fig. 4). To analyze the attainable 346 diversity order, we note the pairwise error probability (PEP) for
SM scales with the Euclidean distance between constellation 347 points as [7]

$$
\operatorname{PEP}\left(\mathbf{y}_{i}, \mathbf{y}_{j}\right)=\mathcal{Q}\left(\sqrt{\frac{\left\|\mathbf{y}_{i}-\mathbf{y}_{j}\right\|^{2}}{2 \sigma^{2}}}\right)
$$

where $\mathcal{Q}(x)$ denotes the Gaussian Q-function [25], and

$$
\begin{aligned}
\left\|\mathbf{y}_{i}-\mathbf{y}_{j}\right\| & =\sqrt{\left\|\mathbf{y}_{i}\right\|^{2}+\left\|\mathbf{y}_{j}\right\|^{2}-2 \mathbf{y}_{i} \bullet \mathbf{y}_{j}} \\
& =\sqrt{\left\|\mathbf{y}_{i}\right\|^{2}+\left\|\mathbf{y}_{j}\right\|^{2}-2\left\|\mathbf{y}_{i}\right\|\left\|\mathbf{y}_{j}\right\| \cos (\Delta \phi)}
\end{aligned}
$$

where $\mathbf{a} \bullet \mathbf{b}$ denotes the dot product of vectors, and $\Delta \phi$ denotes 350 the phase difference between the two constellation points. Ac- 351 cordingly, for the purposes of characterizing the diversity order, 352 we define the gain in the MED for the proposed SM-CR as

$$
\begin{aligned}
G(D) & \hat{=} \frac{E\left\{\max _{d} d_{\min }^{d}\right\}}{E\left\{d_{\min }\right\}} \\
& =\frac{E\left\{\max _{d} \min _{m, k}\left\|\mathbf{H} \mathbf{A}_{d} \mathbf{s}_{m_{1}}^{k_{1}}-\mathbf{H} \mathbf{A}_{d} \mathbf{s}_{m_{2}}^{k_{2}}\right\|^{2}\right\}}{E\left\{\min _{m, k}\left\|\mathbf{H} \mathbf{s}_{m_{1}}^{k_{1}}-\mathbf{H} \mathbf{s}_{m_{2}}^{k_{2}}\right\|^{2}\right\}}
\end{aligned}
$$

where we have used the notation $G(D)$ to suggest that the gain 354 is a function of the size of candidate sets $D$. It will be shown 355 in the results section that this gain also represents the transmit 356 diversity gain attained. The following theorem describes an 357 upper bound of this diversity gain.

358

Theorem 1: For a frequency-flat Rayleigh fading channel 359 $\mathbf{H} \sim \mathcal{C} \mathcal{N}\left(0,(1 / 2) \mathbf{I}_{N_{r}} \oplus \mathbf{I}_{N_{t}}\right)$, the gain in the MED of the 360 proposed $\mathrm{SM}-\mathrm{CR}$ is upper bounded as

361

$G(D) \leq G_{u}=\sum_{k=1}^{D}\left(\begin{array}{l}D \\ k\end{array}\right)(-1)^{k+1} e^{n(k-1)} \frac{E i(-n k, n k)}{E i(-n, n)}$

where $n \hat{=}\left(\begin{array}{c}N_{t} M \\ 2\end{array}\right)$, with $\left(\begin{array}{l}p \\ q\end{array}\right)=p ! / q !(p-q)$ ! denoting the binomial 362 coefficient, with $x$ ! being the factorial function and $\operatorname{Ei}(-n, n) 363$ denoting the generalized exponential integral function [25]. 364

Proof: To simplify the analysis, we shall assume that 365 the distances in the receive constellation are statistically in- 366 dependent. It is shown in Fig. 2 that, strictly speaking, this is 367 not true since the constellation points created by each channel 368 are indeed interdependent through the transmit symbol constel- 369 lation. Nevertheless, we will demonstrate in Fig. 3 that this 370 affordable assumption yields a tight upper bound for the gain. 371 First, regarding the product $\mathbf{H} \mathbf{A}_{d}$, it has been shown in [26] that 372 the product of uncorrelated zero-mean Gaussian variables with 373 variances $\sigma_{1}^{2}, \sigma_{2}^{2}$ is also zero-mean Gaussian with a variance 374 equal to $\sigma_{\Pi}^{2}=\sigma_{1}^{2} \sigma_{2}^{2}$. It is therefore clear that, for a normal- 375 ized transmit constellation, the receive vectors are distributed 376 as $\mathbf{y}_{i} \sim \mathcal{C N}\left(0,1 / 2 \mathbf{I}_{N_{r}}\right)$. Accordingly, $\mathbf{y}_{i}-\mathbf{y}_{j} \sim \mathcal{C N}\left(0, \mathbf{I}_{N_{r}}\right), 377$ and therefore, $z \hat{=}\left\|\mathbf{y}_{i}-\mathbf{y}_{j}\right\|^{2} \sim \mathcal{X}_{2 N_{r}}^{2}$, where $\mathcal{X}_{k}^{2}$ denotes the 378 chi-square distribution with $k$ degrees of freedom [25]. The 379 probability density function (PDF) and cumulative distribution 380 function (CDF) of $z$ are, therefore, given by

$$
\begin{aligned}
& f_{z}(x)=\frac{1}{2^{N_{r}} \Gamma\left(N_{r}\right)} x^{N_{r}-1} e^{-x / 2} \\
& F_{z}(x)=\frac{1}{\Gamma\left(N_{r}\right)} \gamma\left(N_{r}, \frac{x}{2}\right)
\end{aligned}
$$


382 where $\Gamma($.$) and \gamma(.,$.$) denote the Gamma and lower incomplete$ 383 Gamma functions, respectively [25]. Based on the theory of 384 order statistics [27], from the $n \hat{=}\left(\begin{array}{c}N_{t} M \\ 2\end{array}\right)$ distances in the re385 ceive SM constellation (see Fig. 2), the minimum distance is 386 distributed as

$$
\begin{aligned}
f_{d_{\text {min }}}(x) & =n f_{z}(x)\left[1-F_{z}(x)\right]^{n-1} \\
& =\frac{n}{2^{N_{r}} \Gamma\left(N_{r}\right)^{n}} x^{N_{r}-1} e^{-x / 2}\left[\Gamma\left(N_{r}, \frac{x}{2}\right)\right]^{n-1} \\
F_{d_{\text {min }}}(x) & =1-\left(1-F_{z}(x)\right)^{n} \\
& =1-\left[\frac{1}{\Gamma\left(N_{r}\right)} \Gamma\left(N_{r}, \frac{x}{2}\right)\right]^{n}
\end{aligned}
$$

387 where $\Gamma(.,$.$) denotes the upper incomplete Gamma function$ 388 and, as mentioned earlier, it is assumed that all distances in 389 the receive SM constellation are independent. Since $d_{\min }$ is 390 nonnegative, its mean is found as

$$
\begin{aligned}
E\left\{d_{\min }\right\} & =\int_{0}^{\infty}\left[1-F_{d_{\min }}(x)\right] d x \\
& =\int_{0}^{\infty}\left[1-F_{z}(x)\right]^{n} d x .
\end{aligned}
$$

391 Let us now derive the mean of the maximum minimum 392 distance in the receive SM constellation as per the proposed 393 technique. We note that, for the normalized TPS factors in (7), 394 the distribution of $\mathbf{y}_{i}$ remains unchanged. Therefore, the PDF 395 and $\mathrm{CDF}$ of $\tau \hat{=} \max _{\mathbf{A}_{d}} d_{\text {min }}$, when selecting the maximum 396 from $D$, candidates are given as

$$
\begin{aligned}
& f_{\tau}(x)=D f_{d_{\min }}(x) F_{d_{\min }}(x)^{D-1} \\
& F_{\tau}(x)=F_{d_{\min }}(x)^{D} .
\end{aligned}
$$

397 Similarly to the aforementioned calculation, for the mean of $398 \tau \hat{=} \max _{\mathbf{A}_{d}} d_{\min }$, we have

$$
\begin{aligned}
E\{\tau\}=\int_{0}^{\infty}\left\{1-F_{\tau}(x)\right\} d x \\
=\int_{0}^{\infty}\left\{1-F_{d_{\min }}(x)^{D}\right\} d x \\
=\int_{0}^{\infty}\left\{1-\left[1-\left(1-F_{z}(x)\right)^{n}\right]^{D}\right\} d x \\
=\int_{0}^{\infty}\left\{1-\sum_{k=0}^{D}\left(\begin{array}{l}
D \\
k
\end{array}\right)(-1)^{k}\left(1-F_{z}(x)\right)^{n k}\right\} d x \\
=\sum_{k=1}^{D}\left(\begin{array}{l}
D \\
k
\end{array}\right)(-1)^{k+1} \int_{0}^{\infty}\left(1-F_{z}(x)\right)^{n k} d x .
\end{aligned}
$$

399 As stated previously, we have used the binomial expansion $400(1-x)^{m}=\sum_{k=0}^{m}\left(\begin{array}{c}m \\ k\end{array}\right)(-1)^{k} x^{k}$. By substituting (22) and (25) 401 into (16), we arrive at the upper bound for the gain in the MED as

$$
G_{u}\left(N_{r}\right)=\sum_{k=1}^{D}\left(\begin{array}{l}
D \\
k
\end{array}\right)(-1)^{k+1} \frac{\int_{0}^{\infty}\left(1-F_{z}(x)\right)^{n k} d x}{\int_{0}^{\infty}\left(1-F_{z}(x)\right)^{n} d x}
$$

where we have used the notation $G_{u}\left(N_{r}\right)$ to clarify that the 402 upper bound here is a function of $N_{r}$. Finally, it can be shown 403 that $\left(d G_{u}\left(N_{r}\right) / d N_{r}\right) \leq 0$, and therefore, the gain is a mono- 404 tonically decreasing function of the number of RAs. Hence, the 405 gain for the case $N_{r}=1$ provides a global upper bound for all 406 cases of $N_{r}$. Indeed, as it is shown in Fig. 3 and is intuitive, the 407 highest gains can be observed for the single-antenna receiver 408 case, which experiences a diversity of one for conventional SM. 409 For this case, from (18), (19), and (26), we get (17). 410

\section{B. Error Probability Trends}

411

Based on the aforementioned diversity calculations, we can 412 derive the BER performance of the proposed scheme in the 413 high-SNR region. Indeed, SM systems with $N_{r}$ uncorrelated 414 RAs have been shown to experience a unit transmit diversity 415 order and receive diversity order of $N_{r}$. Accordingly, since the 416 proposed scheme attains a transmit diversity order of $G(D)$, the 417 total diversity becomes $\delta=N_{r} G(D)$. The resulting probability 418 of error $P_{e}$ follows the trend

419

$$
P_{e}=\alpha \gamma^{-N_{r} G(D)}
$$

where $\gamma$ is the transmit SNR, $\delta=N_{r} G(D)$ is the diversity 420 order based on the calculations of $G(D)$ in Section IV-A, and 421 $\alpha$ is an arbitrary coefficient. The diversity order $\delta=N_{r} G(D) 422$ accounts for the inherent receive diversity $N_{r}$ in the system and 423 the transmit diversity $G(D)$ induced by the proposed scheme. 424 Clearly, as per the upper bound of Theorem 1 in (17) and the 425 $P_{e}$ trend in (27), a lower bound in the resulting probability of 426 error can be obtained. In the following results, we show that 427 the aforementioned performance trend matches the simulated 428 performance in the high-SNR region.

429

\section{Computational Complexity}

It is clear from the aforementioned discussion that the 431 proposed SM-CR leads to an increase in the computational 432 complexity, with respect to conventional SM, due to the need 433 to compute the MED for all the $D$ candidate scaling factor 434 sets. Here, we analyze the increase in computational complexity 435 at the receiver. We later use this analysis to model the power 436 consumption associated with the required signal processing and 437 compare the proposed SM-CR with conventional SM, in terms 438 of the overall power efficiency of transmission. For reference, 439 we have assumed an LTE Type 2 TDD frame structure [28]. 440 This has a 10-ms duration, which consists of 10 subframes, out 441 of which five subframes, containing 14 symbol time slots each, 442 are used for downlink transmission yielding a block size of 443 $B=70$ for the downlink, while the rest are used for both uplink 444 and control information transmission. A slow-fading channel is 445 assumed, where the channel remains constant for the duration 446 of the frame. In Table I, we summarize the computationally 447 dominant operations involved at the receiver for both SM and 448 SM-CR. In these calculations, we have used the fact that the 449 calculation of the norm of a vector with $n$ elements involves 450 $2 n$ elementary operations. In addition, it can be seen that 451 the product $\mathbf{A}_{d} \mathbf{s}_{m}^{k}$ is a scalar that involves a single complex- 452 valued multiplication, and its multiplication with the channel 453 matrix involves an additional $2 N_{r}$ elementary operations per 454 
TABLE I

COMPLEXITY FOR SM AND THE PROPOSED SM-CR SCHEME

\begin{tabular}{|c|c|c|c|c|}
\hline SM-CR & & Operations & SM & Operations \\
\hline \multicolumn{3}{|l|}{ Constellation Optimization } & \multicolumn{2}{|l|}{ Constellation Calculation } \\
\hline $\mathbf{H} \mathbf{A}_{d} \mathbf{s}_{m}^{k}, \forall m, k$ & $\times D$ & $\left(2 N_{r}+1\right) N_{t} M D$ & $\mathbf{H} \mathbf{s}_{m}^{k}, \forall m, k$ & $\left(2 N_{r}+1\right) N_{t} M$ \\
\hline $\begin{array}{l}\mathbf{f}_{m_{1}, m_{2}}^{k_{1}, k_{2}(d)}=\left\|\mathbf{H} \mathbf{A}_{d} \mathbf{s}_{m_{1}}^{k_{1}}-\mathbf{H} \mathbf{A}_{d} \mathbf{s}_{m_{2}}^{k_{2}}\right\| \\
\forall m_{1}, m_{2}, k_{1}, k_{2}, m_{1} \neq m_{2}, k_{1} \neq k_{2}\end{array}$ & $\times D$ & $2 N_{r}\left(\begin{array}{c}N_{t} M \\
2\end{array}\right) D$ & & \\
\hline$d_{m i n}^{(d)}=\min \left\{\mathbf{f}_{m_{1}, m_{2}}^{k_{1}, k_{2}(d)}\right\}$ & $\times D$ & $\left(\begin{array}{c}N_{t} M \\
2\end{array}\right) D$ & & \\
\hline $\mathbf{A}_{o}=\arg \max d_{\min }^{(d)}$ & & $D$ & & \\
\hline \multicolumn{5}{|l|}{ ML Detection } \\
\hline$g_{m}^{k}=\left\|\mathbf{y}-\mathbf{H} \mathbf{A}_{o} \mathbf{s}_{m}^{k}\right\|^{2}, \forall m, k$ & $\times B$ & $2 N_{t} M N_{r} B$ & $g_{m}^{k}=\left\|\mathbf{y}-\mathbf{H} \mathbf{s}_{m}^{k}\right\|^{2}, \forall m, k \quad \times B$ & $2 N_{t} M N_{r} B$ \\
\hline $\arg \min g_{m}^{k}$ & $\times B$ & $N_{t} M B$ & $\arg \min g_{m}^{k}$ & $N_{t} M B$ \\
\hline Total: $\quad(2 N r+1)\left[\left(\begin{array}{c}N_{t} M \\
2\end{array}\right)+N_{t} M\right] I$ & $+D+$ & $\left(2 N_{r}+1\right) N_{t} M B$ & \multicolumn{2}{|c|}{ Total: $\quad\left(2 N_{r}+1\right) N_{t} M(B+1)$} \\
\hline
\end{tabular}

455 constellation point. This has to be done for each of the $N_{t} M$ 456 points in the receive constellation. Accordingly, there are a 457 number of $\left(\begin{array}{c}N_{t} M \\ 2\end{array}\right)$ distances in the constellation, and therefore, 458 there are $\left(\begin{array}{c}N_{t} M \\ 2\end{array}\right)$ norms in the form of (12) that need to be 459 calculated for each candidate scaling factor set. The first three 460 operations in the constellation optimization in Table I need to 461 be done for each candidate set: hence, $D$ times in total. For the $462 \mathrm{ML}$ detection, a number of $N_{t} M$ norms in the form of (13) 463 need to be calculated before the minimum is chosen, and this 464 has to be calculated $B$ times in the frame. Finally, we have 465 used the fact that finding the maximum and the minimum in an $466 n$-element vector requires $n$ operations.

467 Based on the aforementioned calculations, we have the 468 complexities of the SM receiver and of the SM-CR receiver, 469 respectively, in the form of

$$
\begin{aligned}
C_{\mathrm{SM}}(D)= & \left(2 N_{r}+1\right) N_{t} M(B+1) \\
C_{\mathrm{SM}-\mathrm{CR}}(D)= & (2 N r+1)\left[\left(\begin{array}{c}
N_{t} M \\
2
\end{array}\right)+N_{t} M\right] D+D \\
& +(2 N r+1) N_{t} M B
\end{aligned}
$$

470 where it can be seen that the complexity of SM-CR is in the form

$$
C_{\mathrm{SM}-\mathrm{CR}}(D)=\chi D+\psi
$$

471 with

$$
\begin{aligned}
& \chi=(2 N r+1)\left[\left(\begin{array}{c}
N_{t} M \\
2
\end{array}\right)+N_{t} M\right]+1 \\
& \psi=(2 N r+1) N_{t} M B .
\end{aligned}
$$

472 In the following section, we use these expressions to calcu473 late the resulting power consumption related to signal process474 ing at the receiver for the evaluation of the power efficiency of 475 transmission.

\section{6 \\ VI. POWER EFFICIENCY}

477 As the ultimate metric for evaluating the performance478 complexity tradeoff and the overall usefulness of the proposed 479 technique, and toward an energy-efficient communication sys480 tem, we consider the power efficiency of SM-CR compared 481 to SM, as well as its dependence on the number of candidate scaling factor sets $D$. We note that prior studies explore the en- 482 ergy efficiency of SM for the purposes of optimizing the num- 483 ber of antennas employed [30], [31]. Following the modeling in 484 [29] and [32]-[35], we define the transmit power efficiency of 485 the communication link as the bit rate per total transmit power 486 dissipated, i.e., the ratio of the throughput achieved over the 487 consumed power as

$$
\mathcal{P}=\frac{T}{P_{\mathrm{PA}}+\left(1+N_{r}\right) \cdot P_{\mathrm{RF}}+p_{c} \cdot C}
$$

where $P_{\mathrm{PA}}=((\xi / \eta)-1) P$ in watts is the power consumed 489 at the power amplifier to produce the total transmit signal 490 power $P$, with $\eta$ being the power amplifier efficiency and 491 $\xi$ being the modulation-dependent peak-to-average power ratio. 492 Furthermore, $P_{\mathrm{RF}}=P_{\text {mix }}+P_{\text {filt }}+P_{\mathrm{DAC}}$ is the power related 493 to the mixers, to the transmit filters, and to the digital-to-analog 494 converter (DAC), which is assumed constant for the purposes 495 of this work. We use practical values of these from [32] as 496 $\eta=0.35$ and $P_{\text {mix }}=30.3 \mathrm{~mW}, P_{\text {filt }}=2.5 \mathrm{~mW}$, and $P_{\mathrm{DAC}}=497$ $1.6 \mathrm{~mW}$, yielding $P_{\mathrm{RF}}=34.4 \mathrm{~mW}$. In (33), $p_{c}$ in watts/Kops is 498 the power per $10^{3}$ elementary operations (KOps) of the digital 499 signal processor (DSP), and $C$ is the number of operations 500 involved. This term is used to introduce complexity as a factor 501 of the transmitter power consumption in the power efficiency 502 metric. Typical values of $p_{c}$ include $p_{c}=22.88 \mathrm{~mW} / \mathrm{KOps}$ for 503 the Virtex-4 and $p_{c}=5.76 \mathrm{~mW} / \mathrm{KOps}$ for the Virtex-5 field- 504 programmable gate array family from Xilinx [36]. Finally

$$
T=\mathcal{E} B\left(1-P_{B}\right)=\mathcal{E} B\left(1-P_{e}\right)^{B}
$$

represents the achieved throughput, where $P_{B}$ is the block error 506 rate, and

$$
\mathcal{E}=\log _{2}\left(N_{t} M\right)
$$

is the spectral efficiency of SM in bits per channel use. For a 508 given transmit power and numbers of TAs and RAs, combining 509 (33) with (27), the power efficiency expression for SM-CR 510 takes the form

$$
\mathcal{P}=\frac{\mathcal{E} B\left(1-\alpha \gamma^{-N_{r} G(D)}\right)^{B}}{c+p_{c} C(D)}
$$


512 where both $G(D)$ and $C(D)$ are functions of the number 513 of candidate sets $D$ through (26) and (29), while $\alpha, c$ are 514 constants. This expression can therefore be used to characterize 515 the scalable performance-complexity tradeoff for the proposed 516 scheme and for optimizing the value of $D$ for maximizing 517 power efficiency.

518 The expression in (33) provides an amalgamated metric that 519 combines throughput, complexity, and transmit signal power, 520 all in a unified metric. By varying the number of candidate 521 scaling factor sets $D$, both the resulting complexity and trans522 mission rates are influenced, as shown earlier. Therefore, a 523 scalable tradeoff between performance and complexity can be 524 achieved accordingly. High values of $\mathcal{P}$ indicate that high bit 525 rates are achievable for a given power consumption, and thus 526 denote a high energy efficiency. The following results show that 527 SM-CR provides an increased energy efficiency compared to $528 \mathrm{SM}$ in numerous scenarios using different transmit powers $P$.

\section{Simulation Results}

530 To evaluate the benefits of the proposed technique, this 531 section presents numerical results based on Monte Carlo sim532 ulations of conventional SM without scaling (termed as SM in 533 the figures) and the proposed SM-CR. Our focus is on systems 534 where the receiver employs more than one antenna, where the 535 prescaling schemes in [17]-[19] are inapplicable. The channel 536 impulse response is assumed to be perfectly known at the trans537 mitter. Without loss of generality, unless stated otherwise, we 538 assume that the transmit power is restricted to $P=1$. MIMO 539 systems with up to eight TAs employing 4QAM and 16QAM 540 modulation are explored, albeit it is plausible that the benefits 541 of the proposed technique extend to larger scale systems and 542 higher order modulation.

543 First, for reasons of reference, the BER performance of the 544 proposed scheme is compared with the performance of the most 545 relevant techniques in [17] and [19] for the MISO channel, 546 where the latter techniques are applicable. First, we note the 547 performance loss when applying power scaling to the scheme 548 in [17]. Second, while the true strength of the proposed lies in 549 the fact that it applies to MIMO links where the schemes in 550 [17] and [19] are inapplicable, the results here show that the 551 proposed scheme outperforms the conventional techniques in 552 the MISO channel as well.

553 Next, we show the BER performance with increasing trans554 mit SNR for a $(4 \times 2)$-element MIMO employing 4QAM, for 555 various numbers of candidate scaling factor sets $D$, in Fig. 5. 556 The graph includes the performance of SM for the $(4 \times 4)$ 557 element MIMO for reference. It can be seen that the slope of 558 the BER curves increases with increasing $D$, which indicates 559 an increase in transmit diversity order. Indeed, for high values 560 of $D$, the $(4 \times 2)$-element system with SM-CR exhibits the 561 same transmit diversity order as the $(4 \times 4)$-element system 562 with conventional SM. Moreover, as also observed in Fig. 3, 563 when increasing $D$, the gains saturate for higher values, which 564 can also be seen here, where the BER for $D=20$ closely 565 approximates the one for $D=100$.

566 In Fig. 6 the BER versus SNR performance is shown for the $567(4 \times 2),(8 \times 2)$, and $(8 \times 4)$ systems for both SM and SM-CR.

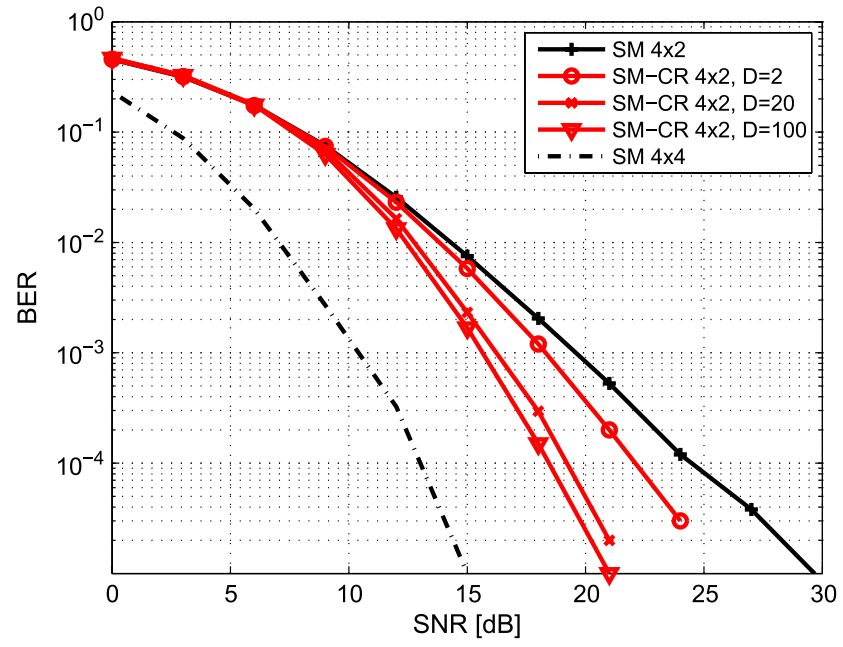

Fig. 5. BER versus SNR for a $(4 \times 2)$ MIMO with $\mathrm{SM}$ and SM-CR with $D=2, D=20$, and $D=100$ for 4QAM.

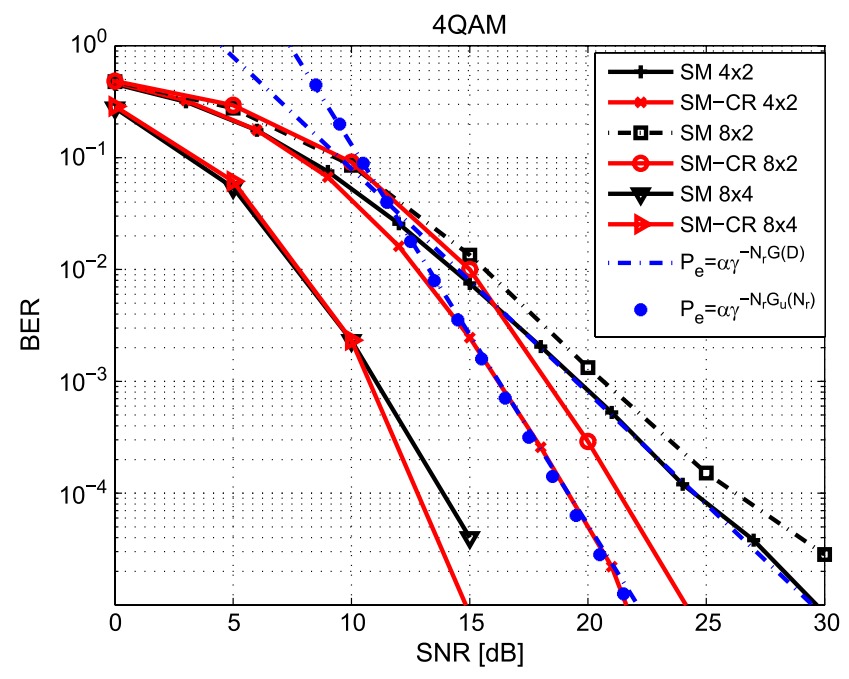

Fig. 6. BER versus SNR for a $(4 \times 2),(8 \times 2)$, and $(8 \times 4)$ MIMO with SM and SM-CR with $D=20$ for 4QAM.

The theoretical diversity trends observed in the form of $P_{e}=568$ $\alpha \gamma^{-\delta}$ are also shown, where $P_{e}$ denotes the probability of error 569 for high SNR; $\gamma$ is the SNR; and $\delta=N_{r} G$ is the diversity order, 570 where $G$ is taken from the respective points in Fig. 3, which is 571 upper bounded, as calculated in Section IV. The performance 572 trends for both the exact diversity gains $G(D)$ based on simu- 573 lation in Fig. 3 and the upper bounds $G_{u}\left(N_{r}\right)$ of Theorem 1 in 574 Section IV-A are shown for comparison. A close match between 575 the analytical and simulated diversity can be observed. With 576 regard to the performance observed, it can be seen that there 577 is indeed a performance penalty when increasing the number 578 of TAs from four to eight for SM with fixed RA number, due 579 to the growth of the spatial constellation, which harms the 580 detection of the TA index [see $(4 \times 2)$ to $(8 \times 2)]$. The improved 581 received diversity in the detection of TA index when increasing 582 the number of RA brings the performance benefits observed in 583 Fig. 6 between $(8 \times 2)$ and $(8 \times 4)$. The same comparison is 584 shown in Fig. 7 for the case of 16QAM, and it can be seen 585 


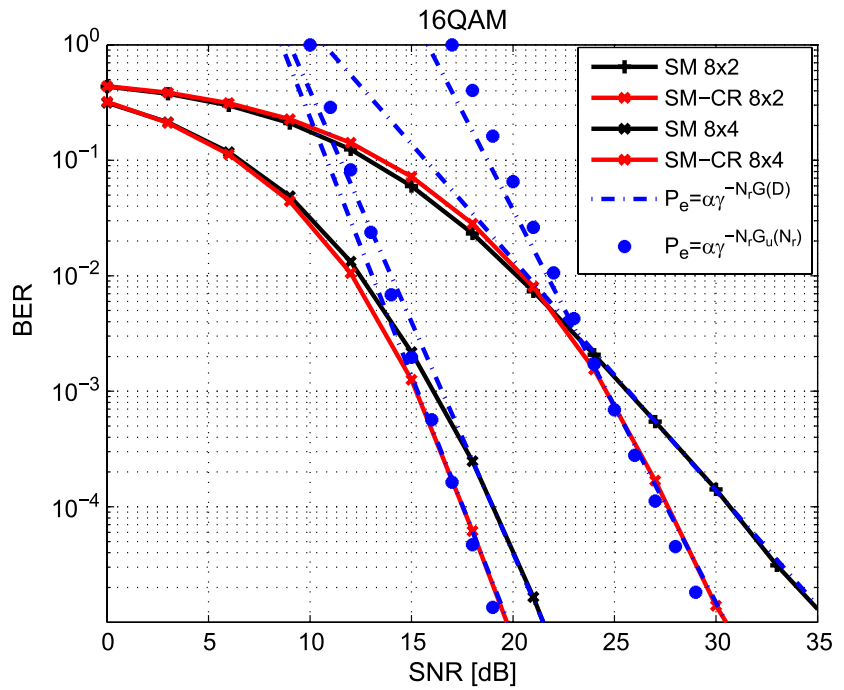

Fig. 7. BER versus SNR for a $(8 \times 2)$ and $(8 \times 4)$ MIMO with SM and SM-CR with $D=20$ for 16QAM.

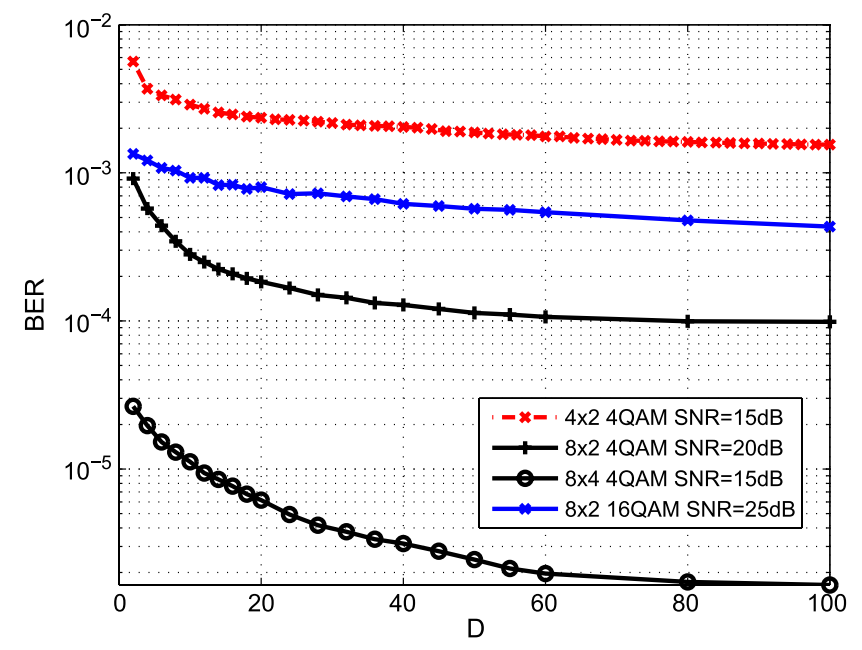

Fig. 8. BER versus $D$ for a $(4 \times 2),(8 \times 2)$, and $(8 \times 4)$ MIMO with SM-CR for $4 \mathrm{QAM}$ and 16QAM.

586 that the performance benefits of the proposed persist. Again, 587 the performance trends for both the exact diversity gains $G(D)$ 588 based on simulation in Fig. 3 and the upper bounds $G_{u}\left(N_{r}\right)$ 589 of Theorem 1 in Section IV-A are shown for comparison. It 590 can be observed that simulation closely matches the theoretical 591 performance trend with both exact diversity gains and their 592 upper bounds, verifying the increase in transmit diversity order, 593 as proven theoretically.

594 Fig. 8 shows the BER as a function of $D$ for the $(4 \times 2)$, $595(8 \times 2)$, and $(8 \times 4)$ with 4 QAM and 16 QAM and various 596 transmit SNR values. Clear gains in the BER can be observed 597 by increasing $D$ in its lower region, while the performance ben598 efits saturate with increasing $D$ in its higher region. Overall, the 599 results illustrate how the theoretically proven gains in transmit 600 diversity translate to improvement in the error performance for 601 the proposed SM-CR.

602 The fact that the scaling factors for the proposed scheme are 603 computed independently at the transmitter and receiver justifies

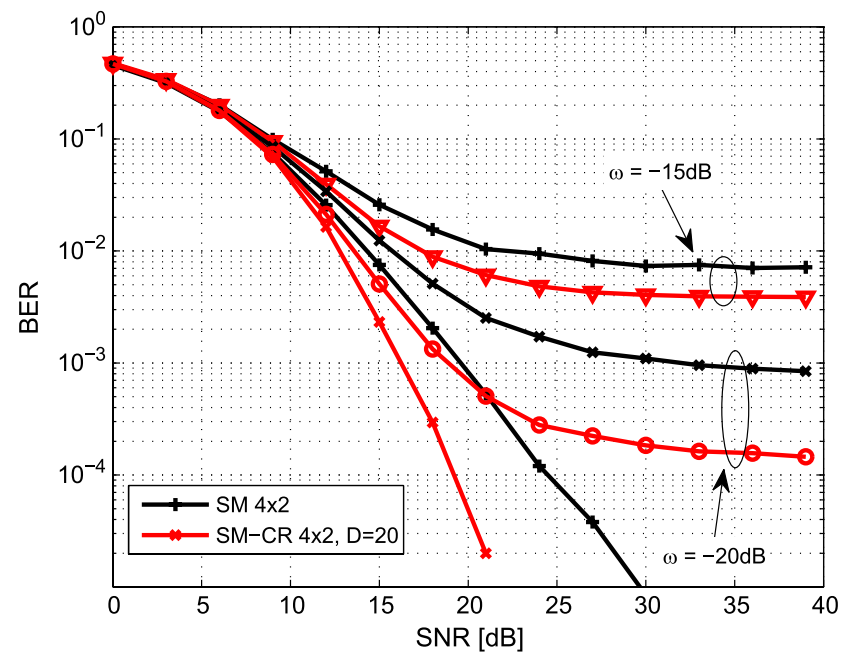

Fig. 9. BER versus SNR for a $(4 \times 2)$ MIMO with CSI errors for SM and SM-CR with $D=20$, for 4QAM.

a study of the performance attainable in the presence of CSI 604 errors and, in particular, in the case where the CSI estimated 605 at the transmitter (CSIT) and the receiver (CSIR) are different. 606 For this reason, in Fig. 9, we explore the situation where both 607 the transmitter (TPS selection) and the receiver (TPS selection 608 and ML detection) rely on erroneous CSI. We model CSIT and 609 CSIR in the form [9]

$$
\hat{\mathbf{H}}=\mathbf{H}+\mathbf{E}
$$

where $\hat{\mathbf{H}}$ and $\mathbf{E} \sim \mathcal{C} \mathcal{N}(0, \omega)$ are the estimated channel and the 611 complex Gaussian CSI error having a variance $\omega$, respectively. 612 Independent CSI error matrices are generated at the transmitter 613 and receiver. Fig. 9 illustrates the BER performance upon 614 increasing the CSIT and CSIR errors for SM and SM-CR, with 615 $\omega$ at $15 \mathrm{~dB}$ and $20 \mathrm{~dB}$ below the signal power. Both techniques 616 are affected by the CSIR errors at the ML detection stage. In 617 addition, for SM-CR, the errors may lead to the selection of dif- 618 ferent TPS factors at the transmitter and receiver. Nonetheless, 619 it can be seen that both SM and SM-CR experience the same 620 performance degradation trend with increasing the CSI errors 621 and that the performance gains observed for SM-CR persist. $\quad 622$

The computational complexity of the proposed technique is 623 examined in Fig. 10, as a function of both $N_{t}$ and $D$, for 4QAM 624 and 16QAM. The complexity count is based on the operations 625 calculated in Table I, and it can be seen that, for both 4QAM 626 and 16QAM, the performance benefits of SM-CR are achieved 627 at an increased complexity compared to SM, which scales with 628 the selection of the parameter $D$. The overall tradeoff between 629 performance and complexity is shown to be favorable for 630 SM-CR in Fig. 11, where the power efficiency is shown with 631 varying transit power for the $(4 \times 2)$ and $(8 \times 2)$ systems with 632 $D=20$. Ranges between $30 \mathrm{dBm}(1 \mathrm{~W})$ and $36 \mathrm{dBm}(4 \mathrm{~W})$ are 633 depicted, which correspond to the power budgets of small-cell 634 base stations [37]. It can be seen that the improved throughput 635 for SM-CR compensates for the increased complexity in the 636 overall system's power efficiency, thus providing an improved 637 tradeoff compared to SM. 

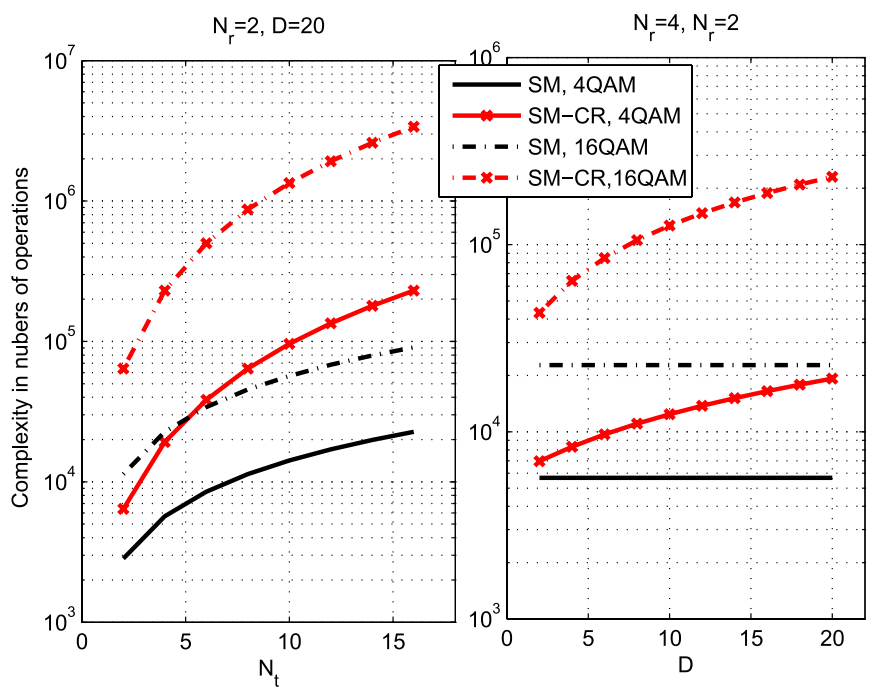

Fig. 10. Computational complexity as a function of $N_{t}$ and $D$ for SM and SM-CR for 4QAM and 16QAM.

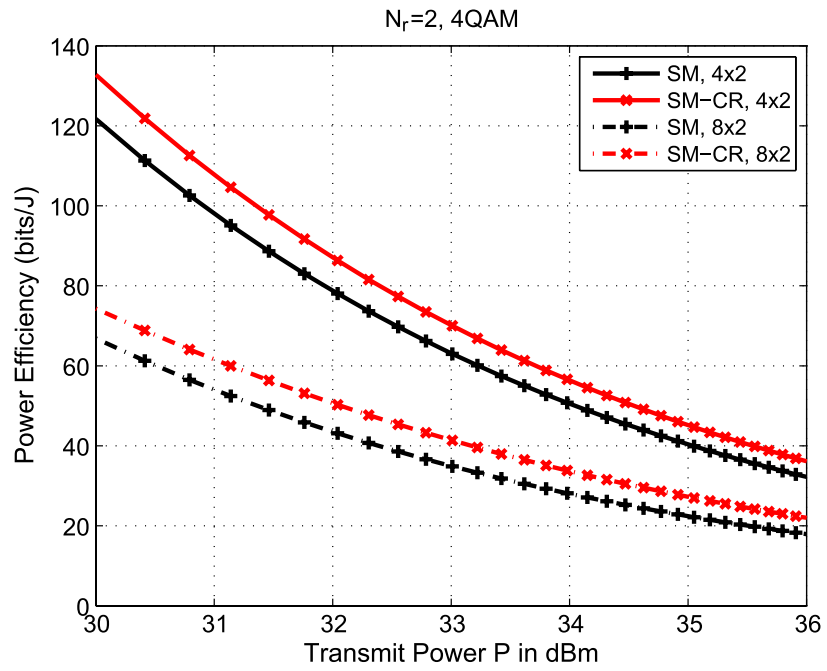

Fig. 11. Power efficiency versus $P$ for a $(4 \times 2)$ and $(8 \times 2)$ MIMO with SM and SM-CR with $D=20$ and $\gamma=18 \mathrm{~dB}$ for 4QAM.

639 Finally, Fig. 12 shows the power efficiency for increasing $D$ 640 for the $(4 \times 2)$ MIMO with transmit SNR $\gamma=15 \mathrm{~dB}$ and the $641(8 \times 2)$ MIMO with $\gamma=20 \mathrm{~dB}$ using 4QAM modulation. The 642 different curves in the figure represent different transmit power 643 budgets ranging from $P=30 \mathrm{dBm}$ to $P=43 \mathrm{dBm}$. For ease 644 of illustration, power efficiency is shown as a percentage of its 645 maximum, as the different scenarios in the figure have different 646 maximum power efficiencies. It can be seen in both subfigures 647 that, as the transmit power is increased, higher values of $D$ offer 648 the best power efficiency. This is due to the fact that, with the 649 increase in the transmit power, the power consumption of the 650 DSP becomes less important and the increase in throughput 651 greatly improves the overall power efficiency. In all cases, the 652 maximum power efficiency achieved with SM-CR is better 653 than the one for conventional SM, which corresponds to the 654 points in the figure with $D=1$, indicating that the proposed 655 scheme offers the required transmission rates at a lower power 656 consumption.
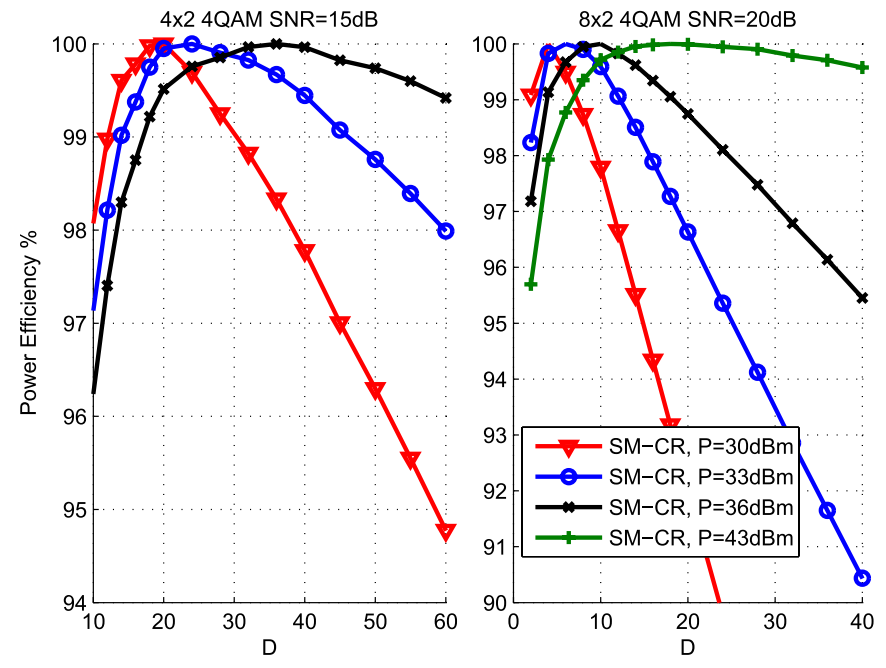

Fig. 12. Power efficiency versus $D$ for a $(4 \times 2),(8 \times 2)$ MIMO with SM and SM-CR for 4QAM.

\section{CONCLUSION}

A new receive-constellation-shaping approach has been intro- 658 duced for SM in the MIMO channel. Conventional constellation- 659 shaping techniques offer limited gains for SM, due to the strict 660 fitting to a fixed constellation, and tend to require the inversion 661 of ill-conditioned channel coefficients. Moreover, existing prac- 662 tical low-complexity constellation-shaping schemes are only ap- 663 plicable to the case where the receiver has a single antenna. We 664 have proposed a CR scheme, where transmit diversity is intro- 665 duced by appropriately selecting the TPS factors from sets of 666 randomly generated coefficients. The proposed scheme has been 667 shown, both analytically and by simulation, to offer significant 668 performance gains with respect to conventional SM. Our future 669 work will involve the application of the proposed approach to 670 more advanced SM techniques, such as generalized SM, as well 671 as SM with antenna selection and adaptive modulation.

\section{REFERENCES}

[1] D. Gesbert, M. Kountouris, R. Heath, C.-B. Chae, and T. Salzer, "Shift- 674 ing the MIMO paradigm," IEEE Signal Process. Mag., vol. 24, no. 5, 675 pp. 36-46, Sep. 2007.

[2] R. Mesleh, H. Haas, S. Sinanovic, C. W. Ahn, and S. Yun, "Spatial 677 modulation," IEEE Trans. Veh. Technol., vol. 57, no. 4, pp. 2228-2241, 678 Jul. 2008

[3] M. Di Renzo and H. Haas, "Bit error probability of space modulation over 680 Nakagami-m fading: Asymptotic analysis," IEEE Commun. Lett., vol. 15, 681 no. 10, pp. 1026-1028, Oct. 2011.

[4] J. Jeganathan, A. Ghrayeb, and L. Szczecinski, "Spatial modulation: 683 Optimal detection and performance analysis," IEEE Commun. Lett., vol. 684 12, no. 8, pp. 545-547, Aug. 2008.

[5] A. Garcia and C. Masouros, "Low-complexity compressive sensing detec- 686 tion for spatial modulation in large-scale multiple access channels," IEEE 687 Trans. Commun., vol. 63, no. 7, pp. 2565-2579, Jul. 2015.

[6] A. Younis, S. Sinanovic, M. Di Renzo, R. Mesleh, and H. Haas, "Gen- 689 eralised sphere decoding for spatial modulation," IEEE Trans. Commun., 690 vol. 61, no. 7, pp. 2805-2815, Jul. 2013.

[7] M. Di Renzo and H. Haas, "On transmit diversity for spatial modulation 692 MIMO: Impact of spatial constellation diagram and shaping filters at the 693 transmitter," IEEE Trans. Veh. Technol., vol. 62, no. 6, pp. 2507-2531, 694 Jul. 2013.

[8] P. Yang et al., "Star-QAM signaling constellations for spatial modulation," 696 IEEE Trans. Veh. Technol., vol. 63, no. 8, pp. 3741-3749, Oct. 2014.697

[9] S. Sugiura, C. Xu, S. X. Ng, and L. Hanzo, "Coherent and differential 698 space-time shift keying: A dispersion matrix approach," IEEE Trans. 699 Commun., vol. 59, no. 11, pp. 3090-3101, Nov. 2011. 
701 [10] K. Ntontin, M. Di Renzo, A. Perez-Neira, and C. Verikoukis, "Adaptive 702 generalized space shift keying," EURASIP J. Wireless Commun. Netw., 703 vol. 2013, pp. 1-15, Feb. 2013.

704 [11] C. Masouros and L. Hanzo, "Dual layered downlink MIMO transmis705 sion for increased bandwidth efficiency," IEEE Trans. Veh. Technol., 706 to be published.

707 [12] C. Masouros and L. Hanzo, "Constructive interference as an information 708

carrier by dual layered MIMO transmission," IEEE Trans. Veh. Technol., DOI: 10.1109/TVT.2015.2438776, to be published.

13] S. Sugiura and L. Hanzo, "On the joint optimization of dispersion matrices and constellations for near-capacity irregular precoded space-time shift keying," IEEE Trans. Wireless Commun., vol. 12, no. 1, pp. 380-387, Jan. 2013.

14] Y. Xiao, Q. Tang, L. Gong, P. Yang, and Z. Yang, "Power scaling for spatial modulation with limited feedback," Int. J. Antennas Propag., vol. 2013, 2013, Art. ID 718482.

15] M. Maleki, H. Bahrami, S. Beygi, M. Kafashan, and N. H. Tran, "Space modulation with CSI: Constellation design and performance evaluation," IEEE Trans. Veh. Technol., vol. 62, no. 4, pp. 1623-1634, May 2013.

6] A. Garcia, C. Masouros, and L. Hanzo, "Pre-scaling optimization for space shift keying based on semidefinite relaxation," IEEE Trans. Commun., vol. 63, no. 11, pp. 4231-4243, Nov. 2015

17] X. Guan, Y. Cai, and W. Yang, "On the mutual information and precoding for spatial modulation with finite alphabet," IEEE Wireless Commun. Lett., vol. 2, no. 4, pp. 383-386, Aug. 2013.

8] J. M. Luna-Rivera, D. U. Campos-Delgado, and M. G. Gonzalez-Perez, "Constellation design for spatial modulation," Procedia Technol., vol. 7, pp. 71-78, 2013.

19] C. Masouros, "Improving the diversity of spatial modulation in MISO channels by phase alignment," IEEE Commun. Lett., vol. 18, no. 5, pp. 729-732, May 2014.

20] P. Yang, M. Di Renzo, Y. Xiao, S. Li, and L. Hanzo, "Design guidelines for spatial modulation," IEEE Commun. Surveys Tuts., vol. 17, no. 1, pp. 6-26, 1st Quart. 2015.

21] M. Di Renzo, H. Haas, A. Ghrayeb, S. Sugiura, and L. Hanzo, "Spatial modulation for generalized MIMO: Challenges, opportunities, and implementation," Proc. IEEE, vol. 102, no. 1, pp. 56-103, Jan. 2014.

22] M. Di Renzo, H. Haas, and P. M. Grant, "Spatial modulation for multipleantenna wireless systems: A survey," IEEE Commun. Mag., vol. 49, no. 12, pp. 182-191, Dec. 2011.

23] J. Jeganathan, A. Ghrayeb, L. Szczecinski, and A. Ceron, "Space shift keying modulation for MIMO channels," IEEE Trans. Wireless Commun., vol. 8, no. 7, pp. 3692-3703, Jul. 2009.

24] M. Di Renzo and H. Haas, "Bit error probability of SM-MIMO over generalized fading channels," IEEE Trans. Veh. Technol., vol. 61, no. 3, pp. 1124-1144, Mar. 2012.

25] M. Abramowitz and I. A. Stegun, Handbook of Mathematical Functions With Formulas, Graphs, and Mathematical Tables. New York, NY, USA: Dover, 1972.

26] R. Ware and F. Lad, "Approximating the distribution for sums of products of normal variables," Univ. Canterbury, Christchurch, New Zealand, Res. Rep., 2003.

27] H. A. David and H. N. Nagaraja, Order Statistics, 3rd ed. New York, NY, USA: Wiley, 2003.

8] Evolved Universal Terrestrial Radio Access (E-UTRA); LTE Physical Layer; General Description, 3GPP TS 36.201, V11.1.0, Rel. 11, Mar. 2008.

29] X. Cong, G. Y. Li, Z. Shunqing, Y. Chen, and S. Xu, "Energy- and spectral-efficiency tradeoff in downlink OFDMA networks," IEEE Trans. Wireless Commun., vol. 10, no. 11, pp. 3874-3886, Nov. 2011.

30] A. Stavridis, S. Sinanovic, M. Di Renzo, and H. Haas, "Energy evaluation of spatial modulation at a multi-antenna base station," in Proc. IEEE 78th VTC-Fall, Sep. 2-5, 2013, pp. 1-5.

31] A. Stavridis, S. Sinanovic, M. Di Renzo, H. Haas, and P. Grant, "An energy saving base station employing spatial modulation," in Proc. IEEE 17th Int. Workshop CAMAD, Sep. 17-19, 2012, pp. 231-235.

32] S. Cui, A. J. Goldsmith, and A. Bahai, "Energy-constrained modulation optimization," IEEE Trans. Wireless Commun., vol. 4, no. 5, pp. 2349-2360, Sep. 2005.

33] C. Masouros, M. Sellathurai, and T. Ratnarajah, "Computationally efficient vector perturbation precoding using thresholded optimization," IEEE Trans. Commun., vol. 61, no. 5, pp. 1880-1890, May 2013.
[34] C. Masouros, M. Sellathurai, and T. Ratnarajah, "Maximizing energy- 773 efficiency in the vector precoded MU-MISO downlink by selective pertur- 774 bation," IEEE Trans. Wireless Commun., vol. 13, no. 9, pp. 4974-4984, 775 Sep. 2014.

[35] C. Masouros, M. Sellathurai, and T. Ratnarajah, "Vector perturbation 777 based on symbol scaling for limited feedback MISO downlinks," IEEE 778 Trans. Signal Process., vol. 62, no. 3, pp. 562-571, Feb. 1, 2014.

[36] D. Curd, "Power consumption in 65 nm FPGAs," Xilinx, San Jose, CA, 780 USA, White Paper, Feb. 2007.

[37] "W-CDMA open access small cells: Architecture, requirements and depen- 782 dencies," Small Cell Forum Ltd, Dursley, U.K., White Paper, May 2012. 783

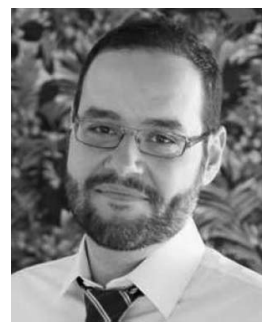

Christos Masouros (M'06-SM'14) received the 784 Diploma in electrical and computer engineering from 785 the University of Patras, Patras, Greece, in 2004 and 786 the M.Sc. degree (by research) and the Ph.D. degree 787 in electrical and electronic engineering from The 788 University of Manchester, Manchester, U.K., in 2006789 and 2009, respectively.

$\mathrm{He}$ is currently a Lecturer with the Department 791 of Electrical and Electronic Engineering, Univer- 792 sity College London, London, U.K. He was previ- 793 ously a Research Associate with The University of 794 Manchester and a Research Fellow with the Queen's University Belfast, Belfast, 795 U.K. His research interests lie in the field of wireless communications and 796 signal processing, with particular focus on green communications, large-scale 797 antenna systems, cognitive radio, and interference mitigation techniques for 798 multiple-input-multiple-output and multicarrier communications.

Dr. Masouros holds a Royal Academy of Engineering Research Fellowship 800 for 2011-2016 and is the Principal Investigator of the Engineering and Physical 801 Sciences Research Council's Project EP/M014150/1 on large-scale antenna sys- 802 tems. He is an Associate Editor for the IEEE COMMUNICATIONS LETTERs. 803

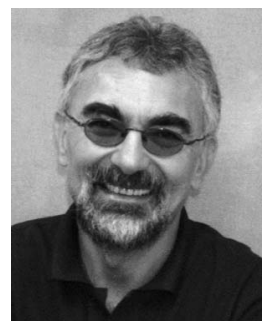

Lajos Hanzo (M'91-SM'92-F'04) received the 804 M.S. degree in electronics and the Ph.D. degree from 805 Budapest University of Technology and Economics 806 (formerly, Technical University of Budapest), 807 Budapest, Hungary, in 1976 and 1983, respectively; 808 the D.Sc. degree from the University of Southampton, 809 Southampton, U.K., in 2004; and the "Doctor Honoris 810 Causa" degree from Budapest University of Technol- 811 ogy and Economics in 2009.

During his 38-year career in telecommunications, 813 he has held various research and academic posts in 814 Hungary, Germany, and the U.K. Since 1986, he has been with the School 815 of Electronics and Computer Science, University of Southampton, where he 816 holds the Chair in Telecommunications. He is currently directing a 100-strong 817 academic research team, working on a range of research projects in the field of 818 wireless multimedia communications sponsored by industry, the Engineering 819 and Physical Sciences Research Council of U.K., the European Research 820 Council's Advanced Fellow Grant, and the Royal Society Wolfson Research 821 Merit Award. During 2008-2012, he was a Chaired Professor with Tsinghua 822 University, Beijing, China. He is an enthusiastic supporter of industrial and 823 academic liaison and offers a range of industrial courses. He has successfully 824 supervised more than $80 \mathrm{Ph} . D$. students, coauthored 20 John Wiley/IEEE Press 825 books on mobile radio communications totaling in excess of 10000 pages, and 826 published more than 1400 research entries on IEEE Xplore. He has more than 827 20000 citations. His research is funded by the European Research Council's 828 Senior Research Fellow Grant.

Dr. Hanzo is a Governor of the IEEE Vehicular Technology Society. He 830 has served as the Technical Program Committee Chair and the General Chair 831 of IEEE conferences, has presented keynote lectures, and has been awarded 832 a number of distinctions. During 2008-2012, he was the Editor-in-Chief of 833 the IEEE Press. He is a Fellow of the Royal Academy of Engineering, The 834 Institution of Engineering and Technology, and the European Association for 835 Signal Processing. 


\section{AUTHOR QUERIES}

\section{AUTHOR PLEASE ANSWER ALL QUERIES}

AQ1 = Please check if the current affiliation of author "Christos Masouros" is properly captured, to be consistent with the biography provided; otherwise, kindly provide the correction.

AQ2 = Please check if Fig. 4 is properly cited here, to properly sequence the citations; otherwise, kindly provide the correction.

AQ3 = Please check if "a factor of" here is properly captured, to make the statement clear; otherwise, kindly provide the correction.

AQ4 = Please check if the expanded form of "FPGA" is properly captured; otherwise, kindly provide the correction.

AQ5 = Please provide publication update in Ref. [12].

\section{END OF ALL QUERIES}




\title{
Constellation Randomization Achieves Transmit Diversity for Single-RF Spatial Modulation
}

\author{
Christos Masouros, Senior Member, IEEE, and Lajos Hanzo, Fellow, IEEE
}

\begin{abstract}
4 Abstract-The performance of spatial modulation (SM) is known 5 to be dominated by the minimum Euclidean distance (MED) in the 6 received SM constellation. In this paper, a symbol-scaling tech7 nique is proposed for SM in the multiple-input-multiple-output 8 (MIMO) channel that enhances the MED to improve the perfor9 mance of SM. This is achieved by forming fixed sets of candidate 10 prescaling factors for the transmit antennas (TAs), which are ran11 domly generated and are known at both the transmitter and the re12 ceiver. For a given channel realization, the transmitter chooses the 13 specific set of factors that maximizes the MED. Given the channel 14 state information (CSI) readily available at the receiver for detec15 tion, the receiver independently chooses the same set of prescaling 16 factors and uses them for the detection of both the antenna index 17 (AI) and the symbol of interest. We analytically calculate the 18 attainable gains of the proposed technique, in terms of its transmit 19 diversity order, based on both the distribution of the MED and on 20 the theory of classical order statistics. Furthermore, we show that 21 the proposed scheme offers a scalable performance-complexity 22 tradeoff for SM by varying the number of candidate sets of pre23 scaling factors, with significant performance improvements, com24 pared to conventional SM.
\end{abstract}

25 Index Terms-Constellation shaping, multiple-input single26 output, prescaling, spatial modulation (SM).

\section{INTRODUCTION}

28

${ }_{30} \mathbf{T}$ RADITIONAL spatial multiplexing has been shown to improve the capacity of the wireless channel by exploiting 31 multiantenna transmitters [1]. More recently, spatial modula32 tion (SM) has been explored as a means of implicitly encoding 33 information in the index of the specific antenna activated for 34 the transmission of the modulated symbols, offering a low35 complexity alternative [2]. Its central benefits include the ab36 sence of interantenna interference (IAI) and the fact that it only 37 requires a subset (down to one) of radio-frequency (RF) chains 38 compared to spatial multiplexing. Accordingly, the interantenna 39 synchronization is also relaxed. Early work has focused on the 40 design of receiver algorithms for minimizing the bit error rate 41 (BER) of SM at a low complexity [2]-[6]. Matched filtering

Manuscript received September 30, 2014; revised June 3, 2015 and November 10, 2015; accepted December 27, 2015. This work was supported by the Royal Academy of Engineering, U.K. The review of this paper was coordinated by Prof. Y. Gong.

C. Masouros is with the Department of Electrical and Electronic Engineering, University College London, London WC1E 7JE, U.K. (e-mail: c.masouros@ ucl.ac.uk)

L. Hanzo is with the School of Electronics and Computer Science, University of Southampton, Southampton SO17 1BJ, U.K. (e-mail: 1h@ecs.soton.ac.uk).

Color versions of one or more of the figures in this paper are available online at http://ieeexplore.iee.org.

Digital Object Identifier 10.1109/TVT.2015.2513380 is shown to be a low-complexity technique for detecting the 42 antenna index (AI) used for SM [2]. A maximum-likelihood 43 (ML) detector is introduced in [4] for reducing the complexity 44 of classic spatial multiplexing ML detectors. Compressive sens- 45 ing and reduced-space sphere detection have been discussed for 46 SM in [5] and [6] for further complexity reduction.

In addition to receive processing, recent work has also pro- 48 posed constellation shaping for SM [7]-[15]. Specifically, in 49 [7], the transmit diversity of coded SM is analyzed for differ- 50 ent spatial constellations, which represent the legitimate sets 51 of activated transmit antennas (TAs). Furthermore, Yang [8] 52 discusses symbol constellation optimization for minimizing the 53 BER. Indeed, spatial- and symbol-constellation shaping are 54 discussed separately, as aforementioned. By contrast, the design 55 of the received SM constellation that combines the choice of 56 the TA, as well as the transmit symbol constellation, is the 57 focus of this paper. Precoding-aided approaches that combine 58 SM with spatial multiplexing are studied in [11] and [12]. A 59 number of constellation-shaping schemes [9]-[15] have also 60 been proposed for the special case of SM, which is referred to 61 as space shift keying, where the information is only carried in 62 the spatial domain, by the activated AI. Their application to the 63 SM transmission, where the transmit waveform is modulated, 64 is nontrivial.

Closely related work has focused on shaping the receive SM 66 constellation by means of symbol prescaling at the transmitter, 67 aiming at maximizing the minimum Euclidean distance (MED) 68 in the received SM constellation [17]-[19]. The constellation- 69 shaping approach in [17] and [18] aims at fitting the receive 70 SM constellation to one of the existing optimal constellation 71 formats in terms of minimum distance, such as, e.g., quadrature 72 amplitude modulation (QAM). Due to the strict constellation 73 fitting requirement imposed on both the amplitude and the 74 phase, this prescaling relies on the inversion of the channel 75 coefficients. In the case of ill-conditioned channels, this sub- 76 stantially increases the power associated to the transmit constel- 77 lation and therefore requires scaling factors for normalizing the 78 transmit power, which, however, reduces the received signal- 79 to-noise ratio (SNR). This problem has been alleviated in [19], 80 where a constellation-shaping scheme based on phase-only 81 scaling is proposed. Nevertheless, the constellation shaping 82 used in the aforementioned schemes is limited in the sense that 83 it only applies to multiple-input-single-output (MISO) systems 84 where a single symbol is received for each transmission, and 85 thus, the characterization and shaping of the receive SM con- 86 stellation is simple. The application of constellation shaping in 87 the multiple-input-multiple-output (MIMO) systems is still an 88 open problem. 


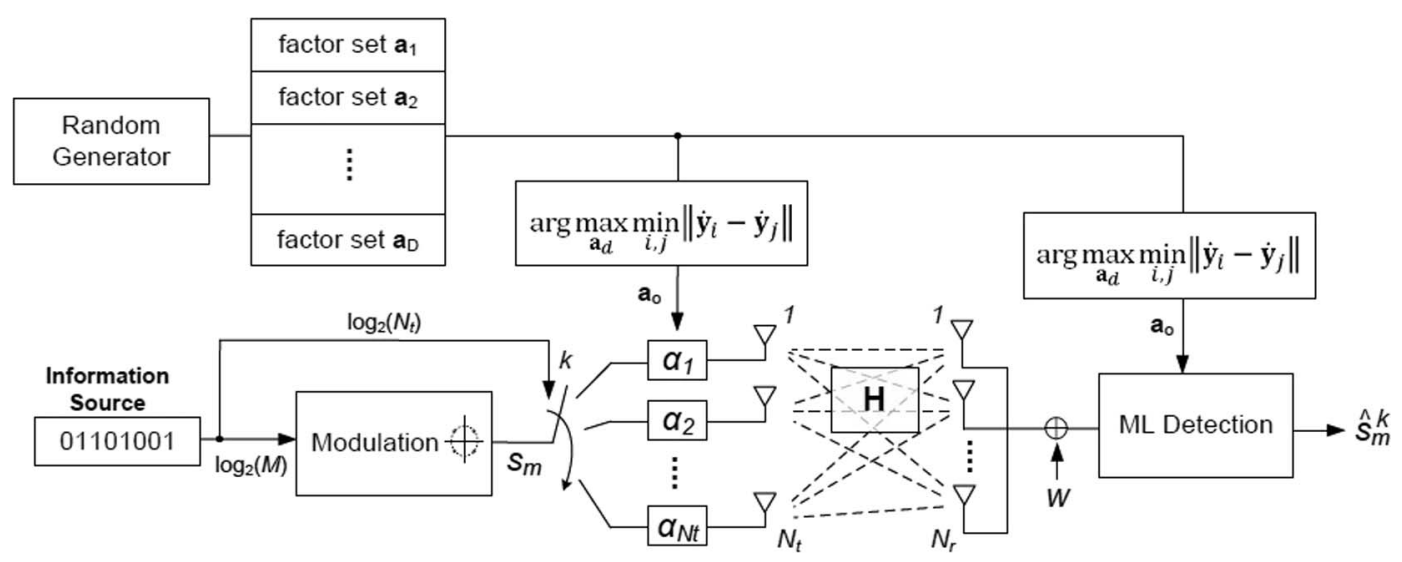

Fig. 1. Block diagram of SM transceiver with constellation randomization (SM-CR).

90 In line with the aforementioned challenges, in this paper, we 91 introduce a new transmit prescaling (TPS) scheme, where the 92 received constellation fitting problem is relaxed. As opposed 93 to the aforementioned strict constellation fitting approaches, 94 here, the received SM constellation is randomized by TPS for 95 maximizing the MED between its points for a given channel. 96 In more detail, a number of randomly generated candidate sets 97 of TPS factors are formed offline, which are known to both the 98 transmitter and the receiver. Each of these sets is normalized, 99 so that the average transmit power remains unchanged, and 100 yields a different receive constellation for a certain channel 101 realization. For a given channel, the transmitter then selects that 102 particular set of TPS factors that yields the SM constellation 103 having the maximum MED. By doing so, the TPS alleviates 104 the cases where different TAs yield similar received symbols 105 and thus improves the reliability of symbol detection. At the 106 receiver, by exploiting the channel state information (CSI) 107 readily available for detection, the detector selects the same set 108 of TPS factors to form the received constellation and applies 109 an ML test to estimate the data. The explicit benefit of the 110 aforementioned methodology is that it extends the idea of re111 ceive SM constellation shaping to the MIMO scenarios having 112 multiple antennas at the receiver, and it will be shown that it 113 introduces additional transmit diversity gains and improves the 114 power efficiency of the SM system. Against this background, $11 \overline{\text { }}$ we list the main contributions of this paper as follows.
- We propose a new per-antenna TPS scheme for SM-aided point-to-point MIMO transmission that improves the attainable performance.

- We analytically derive a tight upper bound of the transmit diversity gains obtained by the proposed technique, based on the distribution of the MED in the received constellation for transmission over a frequency-flat Rayleigh distributed channel.

- We analyze the computational complexity of the proposed scheme to demonstrate how a scalable performancecomplexity tradeoff can be provided by the proposed technique, when adapting the number of candidate sets of TPS factors.

- Using the aforementioned performance and complexity analyses, we study the power efficiency of the proposed scheme in comparison to conventional SM. We introduce a power efficiency metric that combines the transmit 133 power, the achieved throughput, and the computational 134 complexity imposed to quantify the improved power ef- 135 ficiency offered by the proposed scheme.

The remainder of this paper is organized as follows. Section II 137 presents the MIMO system model and introduces the SM trans- 138 mission. Section III details the proposed TPS scheme, while 139 in Section IV, we present our analytical study of the obtained 140 transmit diversity gains of the proposed scheme. Sections V 141 and VI detail the complexity calculation and the study of the 142 attainable power efficiency. Section VII presents our numerical 143 results, and finally, our conclusions are offered in Section VIII. 144

\section{System Model and Spatial Modulation}

\section{A. System Model}

Consider a MIMO system where the transmitter and receiver 147 are equipped with $N_{t}$ and $N_{r}$ antennas, respectively. For sim- 148 plicity, unless stated otherwise, in this paper, we assume that 149 the transmit power budget is limited to unity, i.e., $P=1$. See 150 [20]-[22] for extensive reviews and tutorials on the basics and 151 state-of-the-art on SM. Here, we focus on the single-RF-chain 152 SM approach, where the transmit vector is in the all-but-one 153 zero form $\mathbf{s}_{m}^{k}=\left[0, \ldots, s_{m}, \ldots, 0\right]^{T}$, where the notation [.] $]^{T} 154$ denotes the transpose operator. Here, $s_{m}, m \in\{1, \ldots, M\}$ is 155 a symbol taken from an $M$-order modulation alphabet that 156 represents the transmitted waveform in the baseband domain 157 conveying $\log _{2}(M)$ bits, and $k$ represents the index of the ac- 158 tivated TA (the index of the nonzero element in $\mathbf{s}_{m}^{k}$ ) conveying 159 $\log _{2}\left(N_{t}\right)$ bits in the spatial domain. Clearly, since $\mathbf{s}$ is an all- 160 zero vector apart from $s_{m}^{k}$, there is no IAI.

The per-antenna TPS approach, which is the focus of this 162 paper, is shown in Fig. 1. The signal fed to each TA is scaled by 163 a complex-valued coefficient $\alpha_{k}, k \in\left\{1, \ldots, N_{t}\right\}$, for which 164 we have $E\left\{\left|\alpha_{k}\right|\right\}=1$, where $|x|$ denotes the amplitude of a 165 complex number $x$, and $E\{$.$\} denotes the expectation operator. 166$ Defining the MIMO channel vector as $\mathbf{H}$, with elements $h_{i, j} 167$ representing the complex channel coefficient between the $i$ th 168 TA to the $j$ th receive antenna (RA), the received symbol vector 169 can be written as

$$
\mathbf{y}=\mathbf{H A} \mathbf{s}_{m}^{k}+\mathbf{w}
$$


171 where $\mathbf{w} \sim \mathcal{C} \mathcal{N}\left(0, \sigma^{2} \mathbf{I}\right)$ is the additive white Gaussian noise 172 component at the receiver, with $\mathcal{C N}\left(\mu, \sigma^{2}\right)$ denoting the cir173 cularly symmetric complex Gaussian distribution with mean $\mu$ 174 and variance $\sigma^{2}$. Furthermore, $\mathbf{A}=\operatorname{diag}(\mathbf{a}) \in \mathbb{C}^{N_{t} \times N_{t}}$ is the 175 TPS matrix with $\mathbf{a}=\left[\alpha_{1}, \alpha_{2}, \ldots, \alpha_{N_{t}}\right]$, and $\operatorname{diag}(\mathbf{x})$ represents 176 the diagonal matrix with its diagonal elements taken from 177 vector $\mathbf{x}$. Note that the diagonal structure of $\mathbf{A}$ guarantees 178 having a transmit vector $\mathbf{t}=\mathbf{A s}$ with a single nonzero element, 179 so that the single-RF-chain aspect of SM is preserved.

180 At the receiver, a joint ML detection of both the TA index 181 and the transmit symbol is obtained by the minimization

$$
\begin{aligned}
{\left[\hat{s}_{m}, \hat{k}\right] } & =\arg \min _{i}\left\|\mathbf{y}-\mathbf{y}_{i}\right\| \\
& =\arg \min _{m, k}\left\|\mathbf{y}-\mathbf{H A} \mathbf{A} \mathbf{s}_{m}^{k}\right\|
\end{aligned}
$$

182 where $\|\mathbf{x}\|$ denotes the norm of vector $\mathbf{x}$, and $\mathbf{y}_{i}$ is the $i$ th con183 stellation point in the received SM constellation. By exploiting 184 the specific structure of the transmit vector, this can be further 185 simplified to

$$
\left[\hat{s}_{m}, \hat{k}\right]=\arg \min _{m, k}\left\|\mathbf{y}-\mathbf{h}_{k} \alpha_{m}^{k} s_{m}\right\|
$$

186 where $\mathbf{h}_{k}$ denotes the $k$ th column of matrix $\mathbf{H}$, and $\alpha_{m}^{k}$ is the 187 TPS coefficient of the $k$ th TA. It is widely recognized that the 188 performance of the detection, as explained earlier, is dominated 189 by the MED between adjacent constellation points $\dot{\mathbf{y}}_{i}, \dot{\mathbf{y}}_{j}$ in the 190 receive SM constellation, i.e.,

$$
d_{\min }=\min _{i, j}\left\|\mathbf{y}_{i}-\mathbf{y}_{j}\right\|^{2}, i \neq j .
$$

191 Accordingly, to improve the likelihood of correct detection, 192 constellation-shaping TPS schemes for SM aim at maximizing 193 this MED. The optimum TPS matrix $\mathbf{A}^{*}$ can be found by 194 solving the optimization

$$
\begin{aligned}
& \mathbf{A}^{*}=\arg \max _{\mathbf{A}} \min _{i, j}\left\|\mathbf{y}_{i}-\mathbf{y}_{j}\right\|^{2}, i \neq j \\
& \text { s.t.c. } \quad \operatorname{trace}\left(\mathbf{A}^{* H} \mathbf{A}^{*}\right) \leq P
\end{aligned}
$$

195 and, additionally for single-RF-chain SM, subject to $\mathbf{A}^{*}$ having 196 a diagonal structure. As aforementioned, $\mathbf{A}^{H}$ and trace $(\mathbf{A})$ 197 represent the Hermitian transpose and trace of matrix A, re198 spectively. The aforementioned optimization, however, is an 199 NP-hard problem, which makes finding the TPS factors pro200 hibitively complex and motivates the conception of lower com201 plexity suboptimal techniques.

\section{B. Prescaling for the MISO Channel}

203 In line with the aforementioned discussions, in [17], a 204 prescaling scheme is proposed for the MISO channel. Assum205 ing a channel vector $\mathbf{h}$, the receive SM constellation is fitted to 206 a $Q$-QAM constellation with $Q=N_{t} M$ by choosing

$$
\tilde{\alpha}_{m}^{k}=\frac{q_{(m-1) M+k}\|\mathbf{h}\|}{h_{k} s_{m} \sqrt{N_{t}}}
$$

207 where $q_{i}$ is the $i$ th constellation point in the $Q$-QAM constel208 lation, and the factor $\|\mathbf{h}\| / \sqrt{N_{t}}$ is used for normalizing the 209 receive constellation so that $E\{|q|\}=1$.
We note that, while the scaling in (6) normalizes the receive 210 constellation, it does not normalize the transmit power. There- 211 fore, power-normalized scaling coefficients should be used in 212 the form

$$
\alpha_{m}^{k}=\frac{\tilde{\alpha}_{m}^{k}}{\|\tilde{\mathbf{a}}\|} .
$$

Nevertheless, it can be seen that for ill-conditioned channel 214 coefficients, even for just one of the TAs, this leads to low 215 power-scaling factors $f=1 /\|\tilde{\mathbf{a}}\|$, which limits the obtainable 216 performance. Finally, note that $\alpha_{m}^{k}$ are data dependent for this 217 approach, as evidenced by the index $m$, which does not allow 218 for a fixed per-antenna scaling coefficient, as shown in Fig. 1. 219 Most importantly, the aforementioned strict constellation fitting 220 cannot be extended to systems having multiple RAs, since 221 the inversion of the full channel matrix $\mathbf{H}$ would result in 222 nonzero elements in the transmit vector $\mathbf{t}$, which means that 223 all TAs are used. Therefore, the important benefit of single-RF 224 transmission of SM is lost.

An alternative is shown in [19], again for the MISO channel, 226 where the scaling factors are in the form

$$
\begin{aligned}
\alpha_{k} & =e^{j \varphi_{k}} \\
\varphi_{k} & =\theta_{i}-\vartheta_{k}
\end{aligned}
$$

where $\vartheta_{k}$ is the phase of the $k$ th channel, and $\theta_{i}$ is the $i$ th angle 228 taken from an equally spaced angle arrangement within $[0,2 \pi) 229$ in the form

$$
\theta_{i}=\frac{2 \pi}{N_{t} M}(i-1), i \in\left\{1, \ldots, N_{t}\right\}
$$

In this way, the phases of the points in the receive SM con- 231 stellation become equispaced, hence maintaining a minimum 232 for the Euclidean distances in the constellation.

233

Aside from their individual limitations and the fact that they 234 are suboptimal, the aforementioned prescaling methods are lim- 235 ited by the fact that they apply solely to MISO systems relying 236 on a single RA and cannot be readily extended to the case of 237 MIMO SM transmission, hence lacking receive diversity. $\quad 238$

\section{Proposed Constellation Randomization PRESCALING (SM-CR)}

To alleviate the drawbacks of the aforementioned techniques, 241 we propose an adaptive TPS technique that randomizes the re- 242 ceived SM constellation. The proposed constellation randomiza- 243 tion (CR) simply selects the "best" from a number of randomly 244 generated sets of per-antenna TPS factors, with the aim of im- 245 proving the resulting MED. By allowing the randomization of 246 the amplitude and phase of the effective channel that combines 247 the TPS factor and the channel gains of the TA, the proposed 248 scheme relaxes the constellation optimization problem and 249 facilitates a better solution for the maximization of $d_{\min }$. In ad- 250 dition, through the aforementioned randomization and selection 251 of the appropriate TPS factors, the proposed scheme critically 252 improves the transmit diversity of the SM system, as will 253 be shown analytically in the following section. The proposed 254 scheme involves the steps as analyzed in the following. 


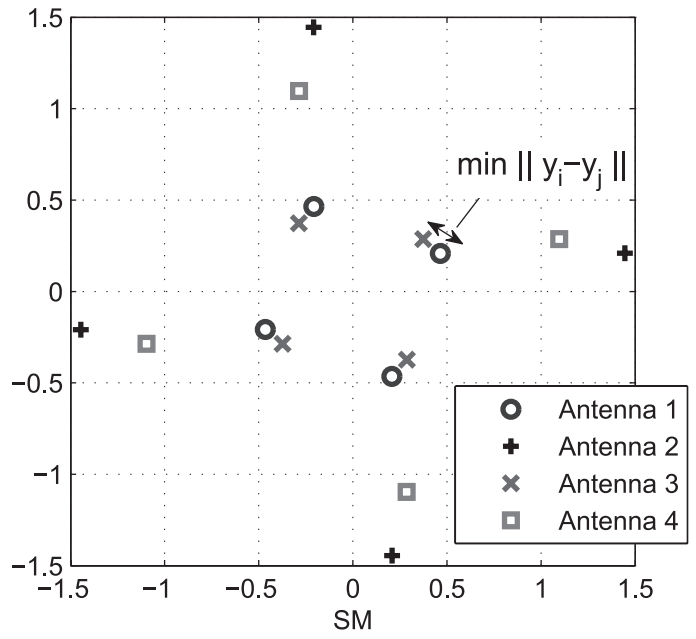

Fig. 2. Received constellation for a $4 \times 1$ MISO with SM and SM-CR for 4QAM.

\section{A. Formation of Candidate Prescaling Sets}

257 First, a number of $D$ candidate TPS vectors are generated 258 randomly in the form $\mathbf{a}_{d}$, where $d \in[1, D]$ denotes the index 259 of the candidate set, and $\mathbf{a}_{d}$ is formed by the elements $\alpha_{m}^{k(d)} \sim$ $260 \mathcal{C N}(0,1)$. These are made available to both the transmitter and 261 the receiver once, in an offline fashion before transmission. 262 These assist in randomizing the received constellation, which 263 is most useful in the cases where two points in the constellation 264 of $\mathbf{H s}_{m}^{k}, m \in[1, M], k \in\left[1, N_{t}\right]$ happen to be very close. To 265 ensure that the average transmit power remains unchanged, the 266 scaling factors are normalized as in (7). It is important to reit267 erate that, in this work, we focus on power-normalized scaling 268 factors, and hence, the proposed scheme does not constitute a 269 power-allocation scheme. This allows us to isolate the diversity 270 gains from the power and coding gains in our analysis in the 271 following section. In the generalized case, power allocation 272 could be applied on top of the prescaling, by employing a 273 diagonal power-allocation matrix, while the resulting diversity 274 gains would not change.

\section{B. Selection of Prescaling Vector}

276 For a given channel, based on the knowledge of vectors $\mathbf{a}_{d}$, 277 both the transmitter and the receiver can determine the received 278 SM constellation for every $d$ by calculating the set of $[m, k]$ 279 possibilities in

$$
\hat{\mathbf{y}}=\mathbf{H} \mathbf{A}_{d} \mathbf{s}_{m}^{k}
$$

280 where $\mathbf{A}_{d}=\operatorname{diag}\left(\mathbf{a}_{d}\right)$ is the diagonal matrix that corresponds 281 to the candidate set $\mathbf{a}_{d}$. Then, for the given channel coefficients, 282 the transmitter and receiver can independently choose the scal283 ing vector $\mathbf{a}_{o}$, for which

$$
\mathbf{a}_{o}=\arg \max _{d} \min _{\substack{m_{1}, m_{2}, k_{1}, k_{2} \\\left\{m_{1}, k_{1}\right\} \neq\left\{m_{2}, k_{2}\right\}}}\left\|\mathbf{H} \mathbf{A}_{d} \mathbf{s}_{m_{1}}^{k_{1}}-\mathbf{H} \mathbf{A}_{d} \mathbf{s}_{m_{2}}^{k_{2}}\right\|^{2} .
$$

284 The transmitter then sends $\mathbf{t}=\mathbf{A}_{o} \mathbf{s}_{m}^{k}$, with $\mathbf{A}_{o}=\operatorname{diag}\left(\mathbf{a}_{o}\right)$, 285 and the receiver applies the ML detector according to

$$
\left[\hat{s}_{m}, \hat{k}\right]=\arg \min _{m, k}\left\|\mathbf{y}-\mathbf{H} \mathbf{A}_{o} \mathbf{s}_{m}^{k}\right\|
$$

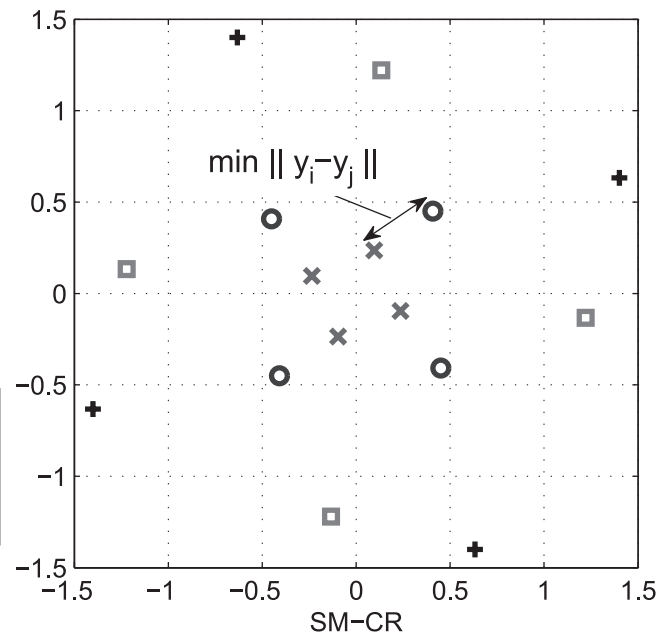

As mentioned earlier, since the channel coefficients are esti- 286 mated at the receiver for detection [2]-[6], (12) can be used to 287 derive the aforementioned factors independently at the receiver. 288 Therefore, no feed forwarding of $\alpha_{m}^{k(d)}$ or the index $d$ is 289 required. Indeed, for equal channel coefficients available at the 290 transmitter and receiver, they both select the same TPS vector 291 $\mathbf{a}_{o}$ independently, as per (12). Alternatively, to dispose of the 292 need for CSI at the transmitter (CSIT), the receiver can indeed 293 select the best scaling factors using (12) and feed the index of 294 the selected scaling vector $\mathbf{a}_{o}$ out of the $D$ candidates back 295 to the transmitter, using $\left\lceil\log _{2} D\right\rceil$ bits. In comparison to the 296 closely related works in [17]-[19], this provides the proposed 297 scheme with the advantage of a reduced transmit complexity 298 that, instead of CSIT acquisition and prescaling optimization, 299 involves the detection of $\left\lceil\log _{2} D\right\rceil$ bits at the end of every 300 channel coherence period, and a single complex multiplication 301 of the classically modulated symbol $s_{m}$ with the prescaling 302 factor $a_{m}^{k}$ in the form shown in (3).

303

The intuitive benefits of the proposed scheme in the MED of 304 the received SM constellation are shown in Fig. 2 for a $(4 \times 1)-305$ element MISO system employing 4QAM modulation at high 306 SNR, where the original receive SM constellation without TPS 307 is shown in the left-hand side, and the constellation after the 308 selection in (12) is illustrated on the right-hand side. A clear 309 increase in the MED can be observed, without increasing the 310 average transmit power. In fact, for the example in Fig. 2, a 311 slight reduction of the power in the symbols denoted by " $x$ " 312 can be observed, which, nevertheless, increases the MED in the 313 constellation.

Observe in Fig. 2 that while suboptimal in the constellation 315 design sense, the proposed TPS enhances the MED in the 316 constellation with respect to conventional SM, while imposing 317 a conveniently scalable complexity as per the size of candidate 318 sets $D$. It is evident that the gains in the MED for the proposed 319 scheme are dependent on the set size $D$ of the candidate 320 TSP vector sets $\mathbf{a}_{d}$ to choose from. An indicative result of 321 this dependence is shown in Fig. 3, where the average gains 322 in the MED are shown, with increasing numbers of $D$ for 323 different transmission scenarios. Theoretically derived upper 324 bounds for these gains for $N_{r}=1, N_{r}=2$, and $N_{r}=4$, based 325 


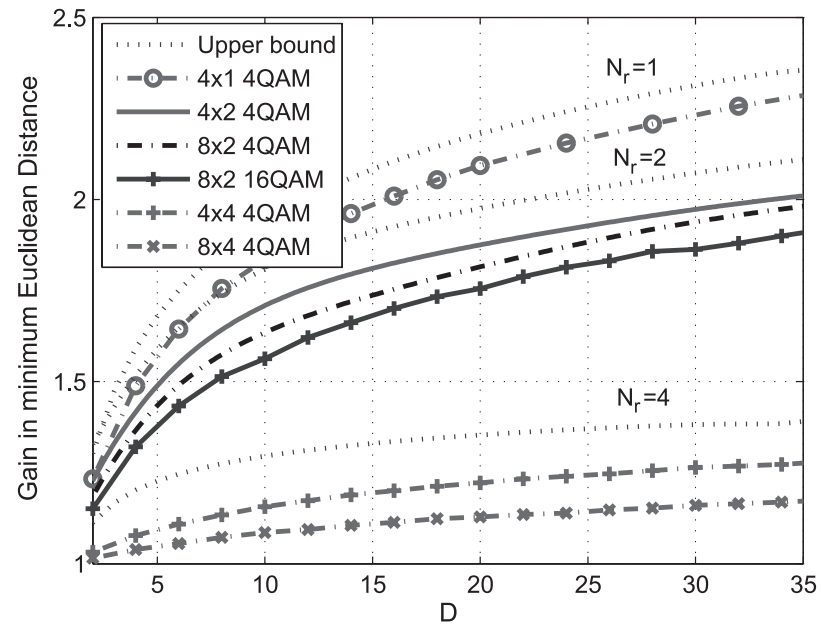

Fig. 3. Gain $G$ in average MED for SM-CR with respect to SM for increasing $D$.

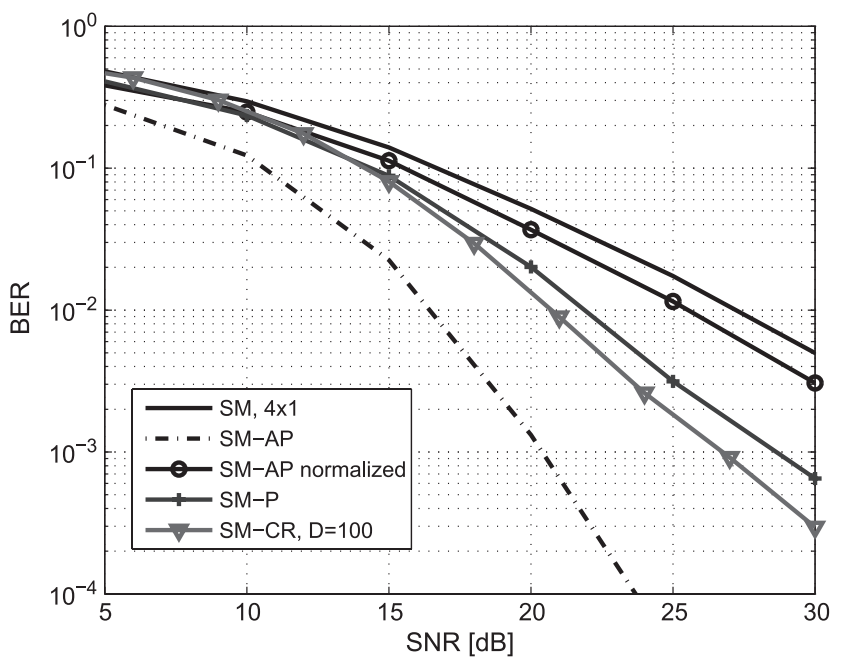

Fig. 4. BER versus SNR for a $(4 \times 1)$ MISO with SM, SM-AP [17], SM-P [19], and SM-CR with $D=100$ for 4QAM.

326 on Theorem 1 of the following section, are also shown in the 327 figure and will be detailed in the following. It can be seen that, 328 for low values of $D$, significant MED benefits are obtained by 329 increasing the number of candidates, while the gains saturate in 330 the region of higher values of $D$. This justifies the choice of low 331 values of $D$ to constrain the computational complexity involved 332 in the search in (12). In the results that follow, we explore the 333 error rates and complexity and their tradeoff in terms of power 334 efficiency as a means of optimizing the value of $D$ for different 335 performance targets.

\section{6 \\ IV. DIVERSITY ANALYSIS}

\section{A. Transmit Diversity}

338 The proposed CR scheme leads to an increase in the transmit 339 diversity gains. That is, while the transmit diversity of the 340 single-RF SM is known to be one [7], the proposed TPS 341 introduces an amplitude-phase diversity in the transmission, 342 due to the existence of $D$ candidate sets of TPS factors from 343 which to choose. The system is said to have a diversity order 344 of $\delta$, if the BER decays with $\gamma^{-\delta}$ in the high-SNR region, 345 with $\gamma$ being the SNR (see Fig. 4). To analyze the attainable 346 diversity order, we note the pairwise error probability (PEP) for
SM scales with the Euclidean distance between constellation 347 points as [7]

$$
\operatorname{PEP}\left(\mathbf{y}_{i}, \mathbf{y}_{j}\right)=\mathcal{Q}\left(\sqrt{\frac{\left\|\mathbf{y}_{i}-\mathbf{y}_{j}\right\|^{2}}{2 \sigma^{2}}}\right)
$$

where $\mathcal{Q}(x)$ denotes the Gaussian Q-function [25], and

$$
\begin{aligned}
\left\|\mathbf{y}_{i}-\mathbf{y}_{j}\right\| & =\sqrt{\left\|\mathbf{y}_{i}\right\|^{2}+\left\|\mathbf{y}_{j}\right\|^{2}-2 \mathbf{y}_{i} \bullet \mathbf{y}_{j}} \\
& =\sqrt{\left\|\mathbf{y}_{i}\right\|^{2}+\left\|\mathbf{y}_{j}\right\|^{2}-2\left\|\mathbf{y}_{i}\right\|\left\|\mathbf{y}_{j}\right\| \cos (\Delta \phi)}
\end{aligned}
$$

where $\mathbf{a} \bullet \mathbf{b}$ denotes the dot product of vectors, and $\Delta \phi$ denotes 350 the phase difference between the two constellation points. Ac- 351 cordingly, for the purposes of characterizing the diversity order, 352 we define the gain in the MED for the proposed SM-CR as

$$
\begin{aligned}
G(D) & \hat{=} \frac{E\left\{\max _{d} d_{\min }^{d}\right\}}{E\left\{d_{\min }\right\}} \\
& =\frac{E\left\{\max _{d} \min _{m, k}\left\|\mathbf{H} \mathbf{A}_{d} \mathbf{s}_{m_{1}}^{k_{1}}-\mathbf{H} \mathbf{A}_{d} \mathbf{s}_{m_{2}}^{k_{2}}\right\|^{2}\right\}}{E\left\{\min _{m, k}\left\|\mathbf{H} \mathbf{s}_{m_{1}}^{k_{1}}-\mathbf{H} \mathbf{s}_{m_{2}}^{k_{2}}\right\|^{2}\right\}}
\end{aligned}
$$

where we have used the notation $G(D)$ to suggest that the gain 354 is a function of the size of candidate sets $D$. It will be shown 355 in the results section that this gain also represents the transmit 356 diversity gain attained. The following theorem describes an 357 upper bound of this diversity gain.

358

Theorem 1: For a frequency-flat Rayleigh fading channel 359 $\mathbf{H} \sim \mathcal{C} \mathcal{N}\left(0,(1 / 2) \mathbf{I}_{N_{r}} \oplus \mathbf{I}_{N_{t}}\right)$, the gain in the MED of the 360 proposed $\mathrm{SM}-\mathrm{CR}$ is upper bounded as

$G(D) \leq G_{u}=\sum_{k=1}^{D}\left(\begin{array}{l}D \\ k\end{array}\right)(-1)^{k+1} e^{n(k-1)} \frac{E i(-n k, n k)}{\operatorname{Ei}(-n, n)}$

where $n \hat{=}\left(\begin{array}{c}N_{t} M \\ 2\end{array}\right)$, with $\left(\begin{array}{l}p \\ q\end{array}\right)=p ! / q !(p-q)$ ! denoting the binomial 362 coefficient, with $x$ ! being the factorial function and $\operatorname{Ei}(-n, n) 363$ denoting the generalized exponential integral function [25]. 364

Proof: To simplify the analysis, we shall assume that 365 the distances in the receive constellation are statistically in- 366 dependent. It is shown in Fig. 2 that, strictly speaking, this is 367 not true since the constellation points created by each channel 368 are indeed interdependent through the transmit symbol constel- 369 lation. Nevertheless, we will demonstrate in Fig. 3 that this 370 affordable assumption yields a tight upper bound for the gain. 371 First, regarding the product $\mathbf{H} \mathbf{A}_{d}$, it has been shown in [26] that 372 the product of uncorrelated zero-mean Gaussian variables with 373 variances $\sigma_{1}^{2}, \sigma_{2}^{2}$ is also zero-mean Gaussian with a variance 374 equal to $\sigma_{\Pi}^{2}=\sigma_{1}^{2} \sigma_{2}^{2}$. It is therefore clear that, for a normal- 375 ized transmit constellation, the receive vectors are distributed 376 as $\mathbf{y}_{i} \sim \mathcal{C} \mathcal{N}\left(0,1 / 2 \mathbf{I}_{N_{r}}\right)$. Accordingly, $\mathbf{y}_{i}-\mathbf{y}_{j} \sim \mathcal{C N}\left(0, \mathbf{I}_{N_{r}}\right), 377$ and therefore, $z \hat{=}\left\|\mathbf{y}_{i}-\mathbf{y}_{j}\right\|^{2} \sim \mathcal{X}_{2 N_{r}}^{2}$, where $\mathcal{X}_{k}^{2}$ denotes the 378 chi-square distribution with $k$ degrees of freedom [25]. The 379 probability density function (PDF) and cumulative distribution 380 function (CDF) of $z$ are, therefore, given by

381

$$
\begin{aligned}
& f_{z}(x)=\frac{1}{2^{N_{r}} \Gamma\left(N_{r}\right)} x^{N_{r}-1} e^{-x / 2} \\
& F_{z}(x)=\frac{1}{\Gamma\left(N_{r}\right)} \gamma\left(N_{r}, \frac{x}{2}\right)
\end{aligned}
$$


382 where $\Gamma($.$) and \gamma(.,$.$) denote the Gamma and lower incomplete$ 383 Gamma functions, respectively [25]. Based on the theory of 384 order statistics [27], from the $n \hat{=}\left(\begin{array}{c}N_{t} M \\ 2\end{array}\right)$ distances in the re385 ceive SM constellation (see Fig. 2), the minimum distance is 386 distributed as

$$
\begin{aligned}
f_{d_{\min }}(x) & =n f_{z}(x)\left[1-F_{z}(x)\right]^{n-1} \\
& =\frac{n}{2^{N_{r}} \Gamma\left(N_{r}\right)^{n}} x^{N_{r}-1} e^{-x / 2}\left[\Gamma\left(N_{r}, \frac{x}{2}\right)\right]^{n-1} \\
F_{d_{\min }}(x) & =1-\left(1-F_{z}(x)\right)^{n} \\
& =1-\left[\frac{1}{\Gamma\left(N_{r}\right)} \Gamma\left(N_{r}, \frac{x}{2}\right)\right]^{n}
\end{aligned}
$$

387 where $\Gamma(.,$.$) denotes the upper incomplete Gamma function$ 388 and, as mentioned earlier, it is assumed that all distances in 389 the receive SM constellation are independent. Since $d_{\min }$ is 390 nonnegative, its mean is found as

$$
\begin{aligned}
E\left\{d_{\min }\right\} & =\int_{0}^{\infty}\left[1-F_{d_{\min }}(x)\right] d x \\
& =\int_{0}^{\infty}\left[1-F_{z}(x)\right]^{n} d x .
\end{aligned}
$$

391 Let us now derive the mean of the maximum minimum 392 distance in the receive SM constellation as per the proposed 393 technique. We note that, for the normalized TPS factors in (7), 394 the distribution of $\mathbf{y}_{i}$ remains unchanged. Therefore, the PDF 395 and $\mathrm{CDF}$ of $\tau \hat{=} \max _{\mathbf{A}_{d}} d_{\min }$, when selecting the maximum 396 from $D$, candidates are given as

$$
\begin{aligned}
f_{\tau}(x) & =D f_{d_{\min }}(x) F_{d_{\min }}(x)^{D-1} \\
F_{\tau}(x) & =F_{d_{\text {min }}}(x)^{D} .
\end{aligned}
$$

397 Similarly to the aforementioned calculation, for the mean of $398 \tau \hat{=} \max _{\mathbf{A}_{d}} d_{\min }$, we have

$$
\begin{aligned}
E\{\tau\}=\int_{0}^{\infty}\left\{1-F_{\tau}(x)\right\} d x \\
=\int_{0}^{\infty}\left\{1-F_{d_{\min }}(x)^{D}\right\} d x \\
=\int_{0}^{\infty}\left\{1-\left[1-\left(1-F_{z}(x)\right)^{n}\right]^{D}\right\} d x \\
=\int_{0}^{\infty}\left\{1-\sum_{k=0}^{D}\left(\begin{array}{l}
D \\
k
\end{array}\right)(-1)^{k}\left(1-F_{z}(x)\right)^{n k}\right\} d x \\
=\sum_{k=1}^{D}\left(\begin{array}{l}
D \\
k
\end{array}\right)(-1)^{k+1} \int_{0}^{\infty}\left(1-F_{z}(x)\right)^{n k} d x .
\end{aligned}
$$

399 As stated previously, we have used the binomial expansion $400(1-x)^{m}=\sum_{k=0}^{m}\left(\begin{array}{c}m \\ k\end{array}\right)(-1)^{k} x^{k}$. By substituting (22) and (25) 401 into (16), we arrive at the upper bound for the gain in the MED as

$$
G_{u}\left(N_{r}\right)=\sum_{k=1}^{D}\left(\begin{array}{l}
D \\
k
\end{array}\right)(-1)^{k+1} \frac{\int_{0}^{\infty}\left(1-F_{z}(x)\right)^{n k} d x}{\int_{0}^{\infty}\left(1-F_{z}(x)\right)^{n} d x}
$$

where we have used the notation $G_{u}\left(N_{r}\right)$ to clarify that the 402 upper bound here is a function of $N_{r}$. Finally, it can be shown 403 that $\left(d G_{u}\left(N_{r}\right) / d N_{r}\right) \leq 0$, and therefore, the gain is a mono- 404 tonically decreasing function of the number of RAs. Hence, the 405 gain for the case $N_{r}=1$ provides a global upper bound for all 406 cases of $N_{r}$. Indeed, as it is shown in Fig. 3 and is intuitive, the 407 highest gains can be observed for the single-antenna receiver 408 case, which experiences a diversity of one for conventional SM. 409 For this case, from (18), (19), and (26), we get (17). 410

\section{B. Error Probability Trends}

411

Based on the aforementioned diversity calculations, we can 412 derive the BER performance of the proposed scheme in the 413 high-SNR region. Indeed, SM systems with $N_{r}$ uncorrelated 414 RAs have been shown to experience a unit transmit diversity 415 order and receive diversity order of $N_{r}$. Accordingly, since the 416 proposed scheme attains a transmit diversity order of $G(D)$, the 417 total diversity becomes $\delta=N_{r} G(D)$. The resulting probability 418 of error $P_{e}$ follows the trend

$$
P_{e}=\alpha \gamma^{-N_{r} G(D)}
$$

where $\gamma$ is the transmit SNR, $\delta=N_{r} G(D)$ is the diversity 420 order based on the calculations of $G(D)$ in Section IV-A, and 421 $\alpha$ is an arbitrary coefficient. The diversity order $\delta=N_{r} G(D) 422$ accounts for the inherent receive diversity $N_{r}$ in the system and 423 the transmit diversity $G(D)$ induced by the proposed scheme. 424 Clearly, as per the upper bound of Theorem 1 in (17) and the 425 $P_{e}$ trend in (27), a lower bound in the resulting probability of 426 error can be obtained. In the following results, we show that 427 the aforementioned performance trend matches the simulated 428 performance in the high-SNR region.

\section{Computational Complexity}

It is clear from the aforementioned discussion that the 431 proposed SM-CR leads to an increase in the computational 432 complexity, with respect to conventional SM, due to the need 433 to compute the MED for all the $D$ candidate scaling factor 434 sets. Here, we analyze the increase in computational complexity 435 at the receiver. We later use this analysis to model the power 436 consumption associated with the required signal processing and 437 compare the proposed SM-CR with conventional SM, in terms 438 of the overall power efficiency of transmission. For reference, 439 we have assumed an LTE Type 2 TDD frame structure [28]. 440 This has a 10-ms duration, which consists of 10 subframes, out 441 of which five subframes, containing 14 symbol time slots each, 442 are used for downlink transmission yielding a block size of 443 $B=70$ for the downlink, while the rest are used for both uplink 444 and control information transmission. A slow-fading channel is 445 assumed, where the channel remains constant for the duration 446 of the frame. In Table I, we summarize the computationally 447 dominant operations involved at the receiver for both SM and 448 SM-CR. In these calculations, we have used the fact that the 449 calculation of the norm of a vector with $n$ elements involves 450 $2 n$ elementary operations. In addition, it can be seen that 451 the product $\mathbf{A}_{d} \mathbf{s}_{m}^{k}$ is a scalar that involves a single complex- 452 valued multiplication, and its multiplication with the channel 453 matrix involves an additional $2 N_{r}$ elementary operations per 454 
TABLE I

COMPleXity For SM AND THE PROPOSED SM-CR SCHEME

\begin{tabular}{|c|c|c|c|c|}
\hline SM-CR & & Operations & SM & Operations \\
\hline \multicolumn{3}{|l|}{ Constellation Optimization } & \multicolumn{2}{|l|}{ Constellation Calculation } \\
\hline $\mathbf{H A}_{d} \mathbf{s}_{m}^{k}, \forall m, k$ & $\times D$ & $\left(2 N_{r}+1\right) N_{t} M D$ & $\mathbf{H} \mathbf{s}_{m}^{k}, \forall m, k$ & $\left(2 N_{r}+1\right) N_{t} M$ \\
\hline $\begin{array}{l}\mathbf{f}_{m_{1}, m_{2}}^{k_{1}, k_{2}(d)}=\left\|\mathbf{H} \mathbf{A}_{d} \mathbf{s}_{m_{1}}^{k_{1}}-\mathbf{H} \mathbf{A}_{d} \mathbf{s}_{m_{2}}^{k_{2}}\right\| \\
\forall m_{1}, m_{2}, k_{1}, k_{2}, m_{1} \neq m_{2}, k_{1} \neq k_{2}\end{array}$ & $\times D$ & $2 N_{r}\left(\begin{array}{c}N_{t} M \\
2\end{array}\right) D$ & & \\
\hline$d_{m i n}^{(d)}=\min \left\{\mathbf{f}_{m_{1}, m_{2}}^{k_{1}, k_{2}(d)}\right\}$ & $\times D$ & $\left(\begin{array}{c}N_{t} M \\
2\end{array}\right) D$ & & \\
\hline $\mathbf{A}_{o}=\arg \max d_{\min }^{(d)}$ & & $D$ & & \\
\hline \multicolumn{5}{|l|}{ ML Detection } \\
\hline$g_{m}^{k}=\left\|\mathbf{y}-\mathbf{H} \mathbf{A}_{o} \mathbf{s}_{m}^{k}\right\|^{2}, \forall m, k$ & $\times B$ & $2 N_{t} M N_{r} B$ & $g_{m}^{k}=\left\|\mathbf{y}-\mathbf{H} \mathbf{s}_{m}^{k}\right\|^{2}, \forall m, k \quad \times B$ & $2 N_{t} M N_{r} B$ \\
\hline $\arg \min g_{m}^{k}$ & $\times B$ & $N_{t} M B$ & $\arg \min g_{m}^{k}$ & $N_{t} M B$ \\
\hline Total: $\quad(2 N r+1)\left[\left(\begin{array}{c}N_{t} M \\
2\end{array}\right)+N_{t} M\right]$ & $+D+$ & $\left(2 N_{r}+1\right) N_{t} M B$ & \multicolumn{2}{|c|}{ Total: $\quad\left(2 N_{r}+1\right) N_{t} M(B+1)$} \\
\hline
\end{tabular}

455 constellation point. This has to be done for each of the $N_{t} M$ 456 points in the receive constellation. Accordingly, there are a 457 number of $\left(\begin{array}{c}N_{t} M \\ 2\end{array}\right)$ distances in the constellation, and therefore, 458 there are $\left(\begin{array}{c}N_{t} M \\ 2\end{array}\right)$ norms in the form of (12) that need to be 459 calculated for each candidate scaling factor set. The first three 460 operations in the constellation optimization in Table I need to 461 be done for each candidate set: hence, $D$ times in total. For the $462 \mathrm{ML}$ detection, a number of $N_{t} M$ norms in the form of (13) 463 need to be calculated before the minimum is chosen, and this 464 has to be calculated $B$ times in the frame. Finally, we have 465 used the fact that finding the maximum and the minimum in an $466 n$-element vector requires $n$ operations.

467 Based on the aforementioned calculations, we have the 468 complexities of the SM receiver and of the SM-CR receiver, 469 respectively, in the form of

$$
\begin{aligned}
C_{\mathrm{SM}}(D)= & \left(2 N_{r}+1\right) N_{t} M(B+1) \\
C_{\mathrm{SM}-\mathrm{CR}}(D)= & (2 N r+1)\left[\left(\begin{array}{c}
N_{t} M \\
2
\end{array}\right)+N_{t} M\right] D+D \\
& +(2 N r+1) N_{t} M B
\end{aligned}
$$

470 where it can be seen that the complexity of SM-CR is in the form

$$
C_{\mathrm{SM}-\mathrm{CR}}(D)=\chi D+\psi
$$

471 with

$$
\begin{aligned}
& \chi=(2 N r+1)\left[\left(\begin{array}{c}
N_{t} M \\
2
\end{array}\right)+N_{t} M\right]+1 \\
& \psi=(2 N r+1) N_{t} M B
\end{aligned}
$$

472 In the following section, we use these expressions to calcu473 late the resulting power consumption related to signal process$474 \mathrm{ing}$ at the receiver for the evaluation of the power efficiency of 475 transmission.

\section{6 \\ VI. POWER EFFICIENCY}

477 As the ultimate metric for evaluating the performance478 complexity tradeoff and the overall usefulness of the proposed 479 technique, and toward an energy-efficient communication sys480 tem, we consider the power efficiency of SM-CR compared 481 to $\mathrm{SM}$, as well as its dependence on the number of candidate scaling factor sets $D$. We note that prior studies explore the en- 482 ergy efficiency of SM for the purposes of optimizing the num- 483 ber of antennas employed [30], [31]. Following the modeling in 484 [29] and [32]-[35], we define the transmit power efficiency of 485 the communication link as the bit rate per total transmit power 486 dissipated, i.e., the ratio of the throughput achieved over the 487 consumed power as

$$
\mathcal{P}=\frac{T}{P_{\mathrm{PA}}+\left(1+N_{r}\right) \cdot P_{\mathrm{RF}}+p_{c} \cdot C}
$$

where $P_{\mathrm{PA}}=((\xi / \eta)-1) P$ in watts is the power consumed 489 at the power amplifier to produce the total transmit signal 490 power $P$, with $\eta$ being the power amplifier efficiency and 491 $\xi$ being the modulation-dependent peak-to-average power ratio. 492 Furthermore, $P_{\mathrm{RF}}=P_{\mathrm{mix}}+P_{\mathrm{filt}}+P_{\mathrm{DAC}}$ is the power related 493 to the mixers, to the transmit filters, and to the digital-to-analog 494 converter (DAC), which is assumed constant for the purposes 495 of this work. We use practical values of these from [32] as 496 $\eta=0.35$ and $P_{\text {mix }}=30.3 \mathrm{~mW}, P_{\text {filt }}=2.5 \mathrm{~mW}$, and $P_{\mathrm{DAC}}=497$ $1.6 \mathrm{~mW}$, yielding $P_{\mathrm{RF}}=34.4 \mathrm{~mW}$. In (33), $p_{c}$ in watts/Kops is 498 the power per $10^{3}$ elementary operations (KOps) of the digital 499 signal processor (DSP), and $C$ is the number of operations 500 involved. This term is used to introduce complexity as a factor 501 of the transmitter power consumption in the power efficiency $502 \mathrm{AQ3}$ metric. Typical values of $p_{c}$ include $p_{c}=22.88 \mathrm{~mW} / \mathrm{KOps}$ for 503 the Virtex-4 and $p_{c}=5.76 \mathrm{~mW} / \mathrm{KOps}$ for the Virtex-5 field- 504 programmable gate array family from Xilinx [36]. Finally

$$
T=\mathcal{E} B\left(1-P_{B}\right)=\mathcal{E} B\left(1-P_{e}\right)^{B}
$$

represents the achieved throughput, where $P_{B}$ is the block error 506 rate, and

$$
\mathcal{E}=\log _{2}\left(N_{t} M\right)
$$

is the spectral efficiency of SM in bits per channel use. For a 508 given transmit power and numbers of TAs and RAs, combining 509 (33) with (27), the power efficiency expression for SM-CR 510 takes the form

$$
\mathcal{P}=\frac{\mathcal{E} B\left(1-\alpha \gamma^{-N_{r} G(D)}\right)^{B}}{c+p_{c} C(D)}
$$


512 where both $G(D)$ and $C(D)$ are functions of the number 513 of candidate sets $D$ through (26) and (29), while $\alpha, c$ are 514 constants. This expression can therefore be used to characterize 515 the scalable performance-complexity tradeoff for the proposed 516 scheme and for optimizing the value of $D$ for maximizing 517 power efficiency.

518 The expression in (33) provides an amalgamated metric that 519 combines throughput, complexity, and transmit signal power, 520 all in a unified metric. By varying the number of candidate 521 scaling factor sets $D$, both the resulting complexity and trans522 mission rates are influenced, as shown earlier. Therefore, a 523 scalable tradeoff between performance and complexity can be 524 achieved accordingly. High values of $\mathcal{P}$ indicate that high bit 525 rates are achievable for a given power consumption, and thus 526 denote a high energy efficiency. The following results show that 527 SM-CR provides an increased energy efficiency compared to $528 \mathrm{SM}$ in numerous scenarios using different transmit powers $P$.

\section{Simulation Results}

530 To evaluate the benefits of the proposed technique, this 531 section presents numerical results based on Monte Carlo sim532 ulations of conventional SM without scaling (termed as SM in 533 the figures) and the proposed SM-CR. Our focus is on systems 534 where the receiver employs more than one antenna, where the 535 prescaling schemes in [17]-[19] are inapplicable. The channel 536 impulse response is assumed to be perfectly known at the trans537 mitter. Without loss of generality, unless stated otherwise, we 538 assume that the transmit power is restricted to $P=1$. MIMO 539 systems with up to eight TAs employing 4QAM and 16QAM 540 modulation are explored, albeit it is plausible that the benefits 541 of the proposed technique extend to larger scale systems and 542 higher order modulation.

543 First, for reasons of reference, the BER performance of the 544 proposed scheme is compared with the performance of the most 545 relevant techniques in [17] and [19] for the MISO channel, 546 where the latter techniques are applicable. First, we note the 547 performance loss when applying power scaling to the scheme 548 in [17]. Second, while the true strength of the proposed lies in 549 the fact that it applies to MIMO links where the schemes in 550 [17] and [19] are inapplicable, the results here show that the 551 proposed scheme outperforms the conventional techniques in 552 the MISO channel as well.

553 Next, we show the BER performance with increasing trans554 mit SNR for a $(4 \times 2)$-element MIMO employing 4QAM, for 555 various numbers of candidate scaling factor sets $D$, in Fig. 5 . 556 The graph includes the performance of SM for the $(4 \times 4)$ 557 element MIMO for reference. It can be seen that the slope of 558 the BER curves increases with increasing $D$, which indicates 559 an increase in transmit diversity order. Indeed, for high values 560 of $D$, the $(4 \times 2)$-element system with SM-CR exhibits the 561 same transmit diversity order as the $(4 \times 4)$-element system 562 with conventional SM. Moreover, as also observed in Fig. 3, 563 when increasing $D$, the gains saturate for higher values, which 564 can also be seen here, where the BER for $D=20$ closely 565 approximates the one for $D=100$.

566 In Fig. 6 the BER versus SNR performance is shown for the $567(4 \times 2),(8 \times 2)$, and $(8 \times 4)$ systems for both SM and SM-CR.

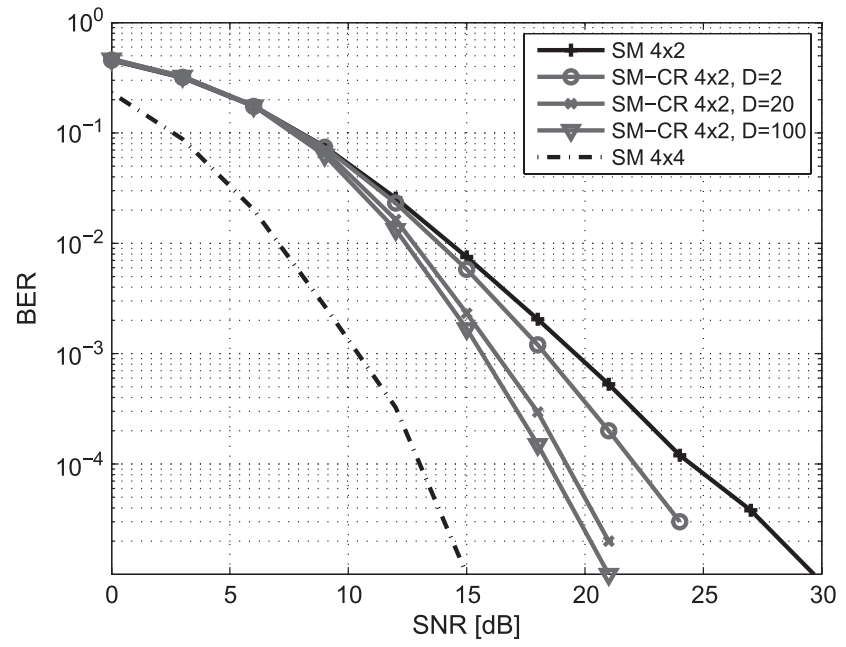

Fig. 5. BER versus SNR for a $(4 \times 2)$ MIMO with SM and SM-CR with $D=2, D=20$, and $D=100$ for 4 QAM.

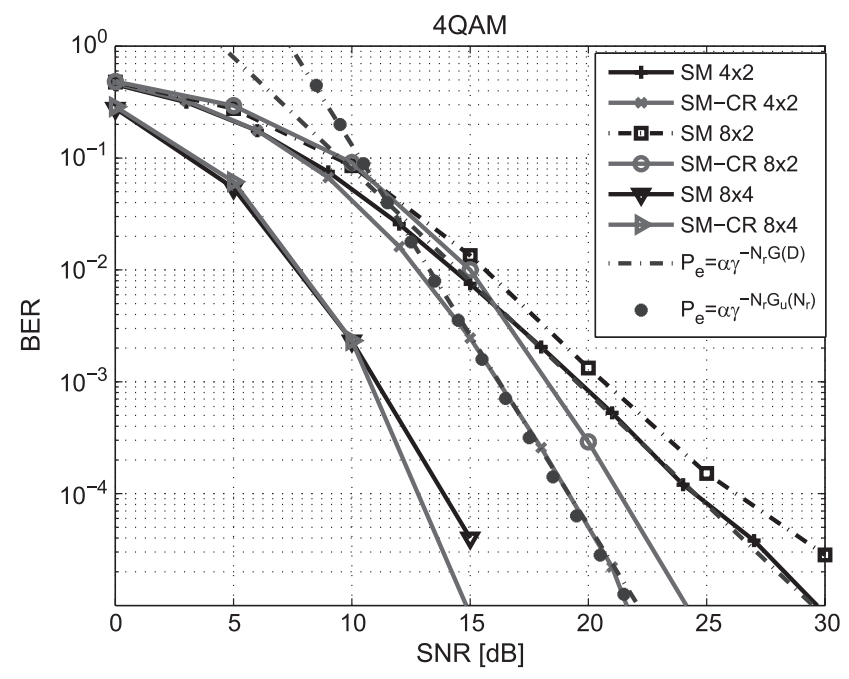

Fig. 6. BER versus SNR for a $(4 \times 2),(8 \times 2)$, and $(8 \times 4)$ MIMO with SM and SM-CR with $D=20$ for 4QAM.

The theoretical diversity trends observed in the form of $P_{e}=568$ $\alpha \gamma^{-\delta}$ are also shown, where $P_{e}$ denotes the probability of error 569 for high SNR; $\gamma$ is the SNR; and $\delta=N_{r} G$ is the diversity order, 570 where $G$ is taken from the respective points in Fig. 3, which is 571 upper bounded, as calculated in Section IV. The performance 572 trends for both the exact diversity gains $G(D)$ based on simu- 573 lation in Fig. 3 and the upper bounds $G_{u}\left(N_{r}\right)$ of Theorem 1 in 574 Section IV-A are shown for comparison. A close match between 575 the analytical and simulated diversity can be observed. With 576 regard to the performance observed, it can be seen that there 577 is indeed a performance penalty when increasing the number 578 of TAs from four to eight for SM with fixed RA number, due 579 to the growth of the spatial constellation, which harms the 580 detection of the TA index [see $(4 \times 2)$ to $(8 \times 2)$ ]. The improved 581 received diversity in the detection of TA index when increasing 582 the number of RA brings the performance benefits observed in 583 Fig. 6 between $(8 \times 2)$ and $(8 \times 4)$. The same comparison is 584 shown in Fig. 7 for the case of 16QAM, and it can be seen 585 


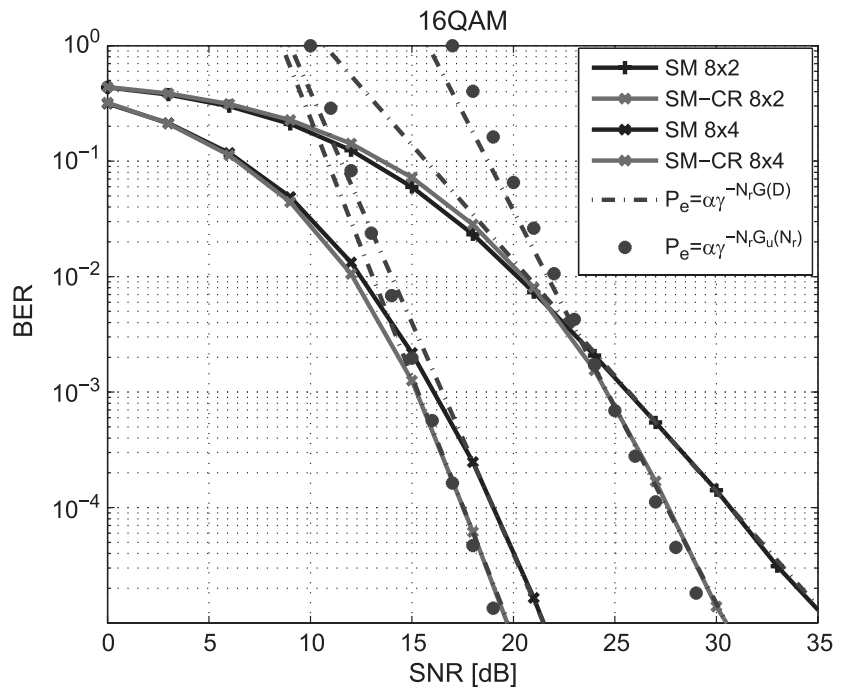

Fig. 7. BER versus SNR for a $(8 \times 2)$ and $(8 \times 4)$ MIMO with SM and SM-CR with $D=20$ for 16QAM.

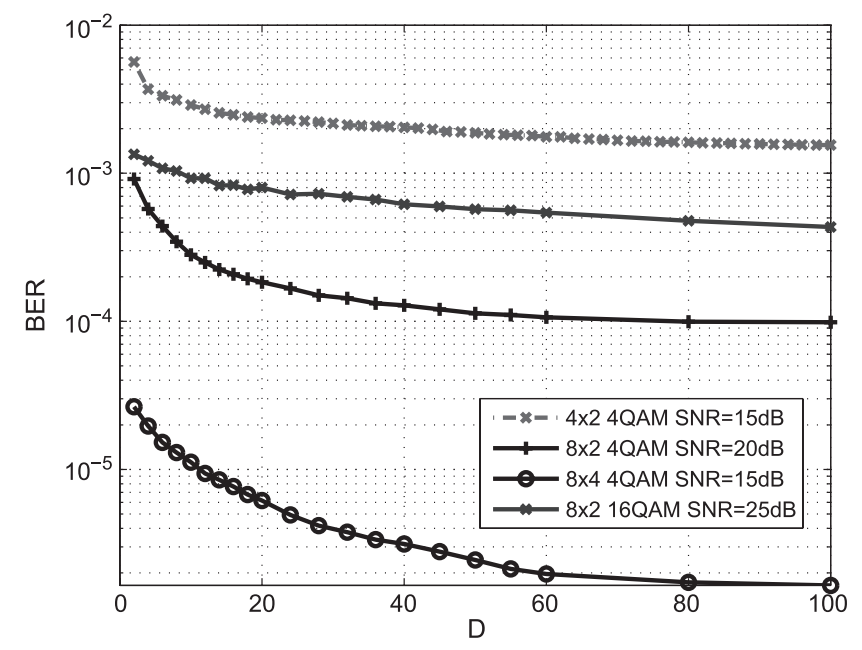

Fig. 8. BER versus $D$ for a $(4 \times 2),(8 \times 2)$, and $(8 \times 4)$ MIMO with SM-CR for 4QAM and 16QAM.

586 that the performance benefits of the proposed persist. Again, 587 the performance trends for both the exact diversity gains $G(D)$ 588 based on simulation in Fig. 3 and the upper bounds $G_{u}\left(N_{r}\right)$ 589 of Theorem 1 in Section IV-A are shown for comparison. It 590 can be observed that simulation closely matches the theoretical 591 performance trend with both exact diversity gains and their 592 upper bounds, verifying the increase in transmit diversity order, 593 as proven theoretically.

594 Fig. 8 shows the BER as a function of $D$ for the $(4 \times 2)$, $595(8 \times 2)$, and $(8 \times 4)$ with 4 QAM and 16 QAM and various 596 transmit SNR values. Clear gains in the BER can be observed 597 by increasing $D$ in its lower region, while the performance ben598 efits saturate with increasing $D$ in its higher region. Overall, the 599 results illustrate how the theoretically proven gains in transmit 600 diversity translate to improvement in the error performance for 601 the proposed SM-CR.

602 The fact that the scaling factors for the proposed scheme are 603 computed independently at the transmitter and receiver justifies

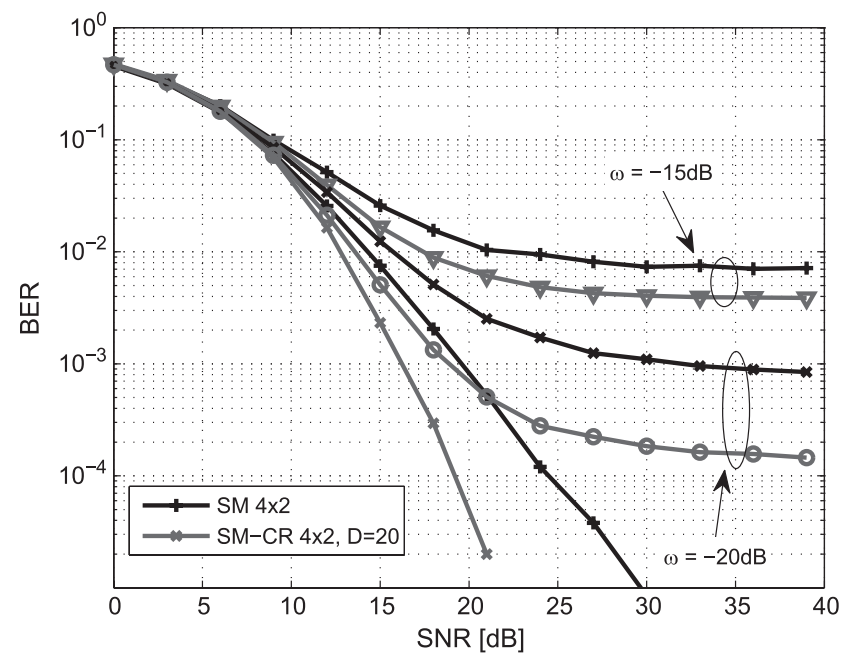

Fig. 9. BER versus SNR for a $(4 \times 2)$ MIMO with CSI errors for SM and SM-CR with $D=20$, for 4QAM.

a study of the performance attainable in the presence of CSI 604 errors and, in particular, in the case where the CSI estimated 605 at the transmitter (CSIT) and the receiver (CSIR) are different. 606 For this reason, in Fig. 9, we explore the situation where both 607 the transmitter (TPS selection) and the receiver (TPS selection 608 and ML detection) rely on erroneous CSI. We model CSIT and 609 CSIR in the form [9]

610

$$
\hat{\mathbf{H}}=\mathbf{H}+\mathbf{E}
$$

where $\hat{\mathbf{H}}$ and $\mathbf{E} \sim \mathcal{C N}(0, \omega)$ are the estimated channel and the 611 complex Gaussian CSI error having a variance $\omega$, respectively. 612 Independent CSI error matrices are generated at the transmitter 613 and receiver. Fig. 9 illustrates the BER performance upon 614 increasing the CSIT and CSIR errors for SM and SM-CR, with 615 $\omega$ at $15 \mathrm{~dB}$ and $20 \mathrm{~dB}$ below the signal power. Both techniques 616 are affected by the CSIR errors at the ML detection stage. In 617 addition, for SM-CR, the errors may lead to the selection of dif- 618 ferent TPS factors at the transmitter and receiver. Nonetheless, 619 it can be seen that both SM and SM-CR experience the same 620 performance degradation trend with increasing the CSI errors 621 and that the performance gains observed for SM-CR persist. $\quad 622$

The computational complexity of the proposed technique is 623 examined in Fig. 10, as a function of both $N_{t}$ and $D$, for 4QAM 624 and 16QAM. The complexity count is based on the operations 625 calculated in Table I, and it can be seen that, for both 4QAM 626 and 16QAM, the performance benefits of SM-CR are achieved 627 at an increased complexity compared to SM, which scales with 628 the selection of the parameter $D$. The overall tradeoff between 629 performance and complexity is shown to be favorable for 630 SM-CR in Fig. 11, where the power efficiency is shown with 631 varying transit power for the $(4 \times 2)$ and $(8 \times 2)$ systems with 632 $D=20$. Ranges between $30 \mathrm{dBm}(1 \mathrm{~W})$ and $36 \mathrm{dBm}(4 \mathrm{~W})$ are 633 depicted, which correspond to the power budgets of small-cell 634 base stations [37]. It can be seen that the improved throughput 635 for SM-CR compensates for the increased complexity in the 636 overall system's power efficiency, thus providing an improved 637 tradeoff compared to SM. 


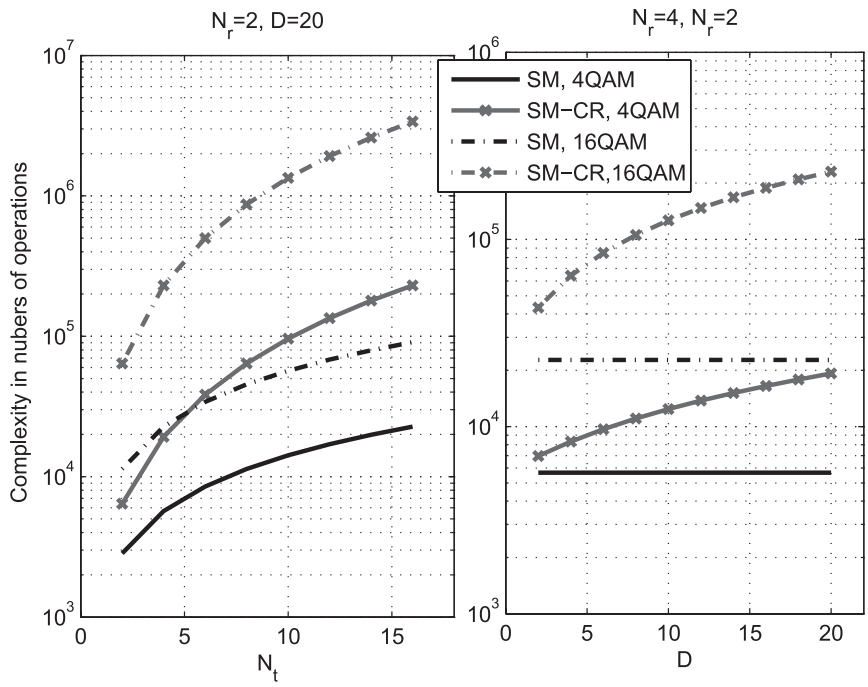

Fig. 10. Computational complexity as a function of $N_{t}$ and $D$ for SM and SM-CR for 4QAM and 16QAM.

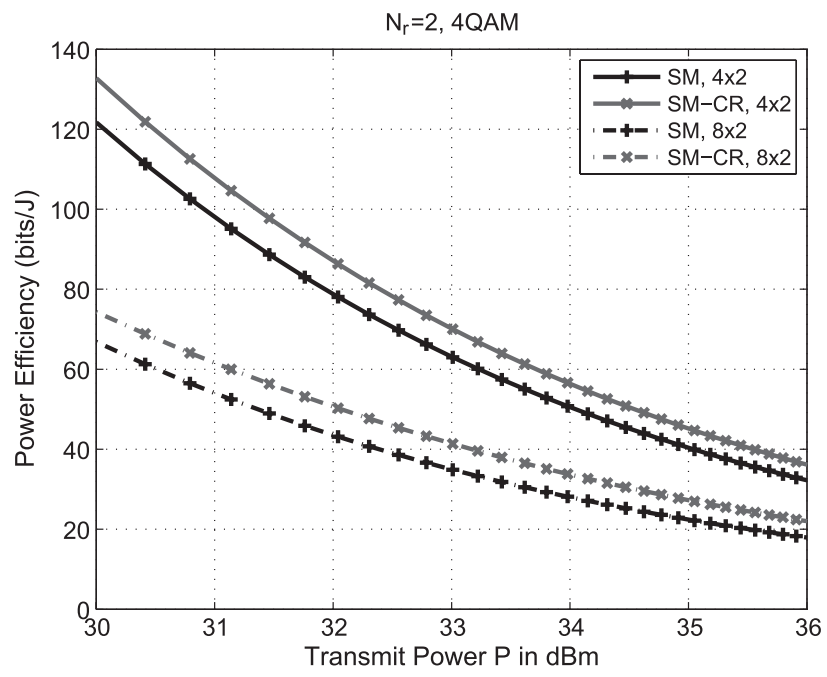

Fig. 11. Power efficiency versus $P$ for a $(4 \times 2)$ and $(8 \times 2)$ MIMO with SM and SM-CR with $D=20$ and $\gamma=18 \mathrm{~dB}$ for 4QAM.

639 Finally, Fig. 12 shows the power efficiency for increasing $D$ 640 for the $(4 \times 2)$ MIMO with transmit SNR $\gamma=15 \mathrm{~dB}$ and the $641(8 \times 2)$ MIMO with $\gamma=20 \mathrm{~dB}$ using 4QAM modulation. The 642 different curves in the figure represent different transmit power 643 budgets ranging from $P=30 \mathrm{dBm}$ to $P=43 \mathrm{dBm}$. For ease 644 of illustration, power efficiency is shown as a percentage of its 645 maximum, as the different scenarios in the figure have different 646 maximum power efficiencies. It can be seen in both subfigures 647 that, as the transmit power is increased, higher values of $D$ offer 648 the best power efficiency. This is due to the fact that, with the 649 increase in the transmit power, the power consumption of the 650 DSP becomes less important and the increase in throughput 651 greatly improves the overall power efficiency. In all cases, the 652 maximum power efficiency achieved with SM-CR is better 653 than the one for conventional SM, which corresponds to the 654 points in the figure with $D=1$, indicating that the proposed 655 scheme offers the required transmission rates at a lower power 656 consumption.
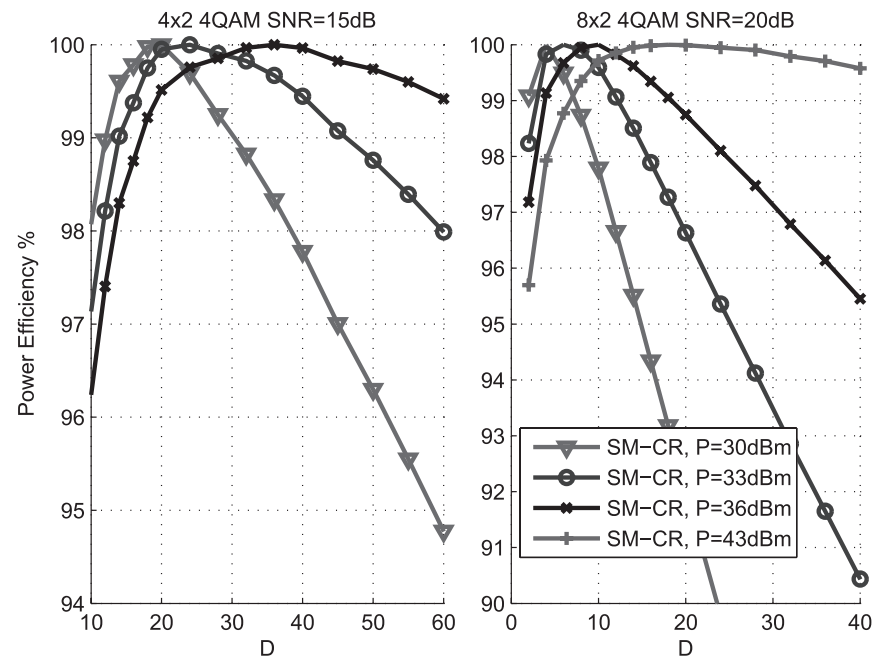

Fig. 12. Power efficiency versus $D$ for a $(4 \times 2),(8 \times 2)$ MIMO with SM and SM-CR for 4QAM.

\section{CONCLUSION}

A new receive-constellation-shaping approach has been intro- 658 duced for SM in the MIMO channel. Conventional constellation- 659 shaping techniques offer limited gains for SM, due to the strict 660 fitting to a fixed constellation, and tend to require the inversion 661 of ill-conditioned channel coefficients. Moreover, existing prac- 662 tical low-complexity constellation-shaping schemes are only ap- 663 plicable to the case where the receiver has a single antenna. We 664 have proposed a CR scheme, where transmit diversity is intro- 665 duced by appropriately selecting the TPS factors from sets of 666 randomly generated coefficients. The proposed scheme has been 667 shown, both analytically and by simulation, to offer significant 668 performance gains with respect to conventional SM. Our future 669 work will involve the application of the proposed approach to 670 more advanced SM techniques, such as generalized SM, as well 671 as $\mathrm{SM}$ with antenna selection and adaptive modulation.

\section{REFERENCES}

[1] D. Gesbert, M. Kountouris, R. Heath, C.-B. Chae, and T. Salzer, "Shift- 674 ing the MIMO paradigm," IEEE Signal Process. Mag., vol. 24, no. 5, 675 pp. 36-46, Sep. 2007.

[2] R. Mesleh, H. Haas, S. Sinanovic, C. W. Ahn, and S. Yun, "Spatial 677 modulation," IEEE Trans. Veh. Technol., vol. 57, no. 4, pp. 2228-2241, 678 Jul. 2008.

[3] M. Di Renzo and H. Haas, "Bit error probability of space modulation over 680 Nakagami-m fading: Asymptotic analysis," IEEE Commun. Lett., vol. 15, 681 no. 10 , pp. 1026-1028, Oct. 2011.

[4] J. Jeganathan, A. Ghrayeb, and L. Szczecinski, "Spatial modulation: 683 Optimal detection and performance analysis," IEEE Commun. Lett., vol. 684 12 , no. 8 , pp. $545-547$, Aug. 2008.

[5] A. Garcia and C. Masouros, "Low-complexity compressive sensing detec- 686 tion for spatial modulation in large-scale multiple access channels," IEEE 687 Trans. Commun., vol. 63, no. 7, pp. 2565-2579, Jul. 2015.

[6] A. Younis, S. Sinanovic, M. Di Renzo, R. Mesleh, and H. Haas, "Gen- 689 eralised sphere decoding for spatial modulation," IEEE Trans. Commun., 690 vol. 61, no. 7, pp. 2805-2815, Jul. 2013.

[7] M. Di Renzo and H. Haas, "On transmit diversity for spatial modulation 692 MIMO: Impact of spatial constellation diagram and shaping filters at the 693 transmitter," IEEE Trans. Veh. Technol., vol. 62, no. 6, pp. 2507-2531, 694 Jul. 2013.

[8] P. Yang et al., "Star-QAM signaling constellations for spatial modulation," 696 IEEE Trans. Veh. Technol., vol. 63, no. 8, pp. 3741-3749, Oct. $2014 . \quad 697$

[9] S. Sugiura, C. Xu, S. X. Ng, and L. Hanzo, "Coherent and differential 698 space-time shift keying: A dispersion matrix approach," IEEE Trans. 699 Commun., vol. 59, no. 11, pp. 3090-3101, Nov. 2011. 
701 [10] K. Ntontin, M. Di Renzo, A. Perez-Neira, and C. Verikoukis, "Adaptive 702 generalized space shift keying," EURASIP J. Wireless Commun. Netw., 703 vol. 2013, pp. 1-15, Feb. 2013.

704 [11] C. Masouros and L. Hanzo, "Dual layered downlink MIMO transmis705 sion for increased bandwidth efficiency," IEEE Trans. Veh. Technol., 706 to be published.

707 [12] C. Masouros and L. Hanzo, "Constructive interference as an information 708 carrier by dual layered MIMO transmission," IEEE Trans. Veh. Technol., 709 DOI: 10.1109/TVT.2015.2438776, to be published.

710 [13] S. Sugiura and L. Hanzo, "On the joint optimization of dispersion matrices 711 and constellations for near-capacity irregular precoded space-time shift 712 keying," IEEE Trans. Wireless Commun., vol. 12, no. 1, pp. 380-387, 713 Jan. 2013

714 [14] Y. Xiao, Q. Tang, L. Gong, P. Yang, and Z. Yang, "Power scaling for 715 spatial modulation with limited feedback," Int. J. Antennas Propag., 716 vol. 2013, 2013, Art. ID 718482.

717 [15] M. Maleki, H. Bahrami, S. Beygi, M. Kafashan, and N. H. Tran, "Space 718 modulation with CSI: Constellation design and performance evaluation," 719 IEEE Trans. Veh. Technol., vol. 62, no. 4, pp. 1623-1634, May 2013.

720 [16] A. Garcia, C. Masouros, and L. Hanzo, "Pre-scaling optimization for 721 space shift keying based on semidefinite relaxation," IEEE Trans. Com722 mun., vol. 63, no. 11, pp. 4231-4243, Nov. 2015

723 [17] X. Guan, Y. Cai, and W. Yang, "On the mutual information and precoding 724 for spatial modulation with finite alphabet," IEEE Wireless Commun. 725 Lett., vol. 2, no. 4, pp. 383-386, Aug. 2013.

726 [18] J. M. Luna-Rivera, D. U. Campos-Delgado, and M. G. Gonzalez-Perez, 727 "Constellation design for spatial modulation," Procedia Technol., vol. 7, $728 \quad$ pp. $71-78,2013$.

729 [19] C. Masouros, "Improving the diversity of spatial modulation in MISO 730 channels by phase alignment," IEEE Commun. Lett., vol. 18, no. 5, 731 pp. 729-732, May 2014.

732 [20] P. Yang, M. Di Renzo, Y. Xiao, S. Li, and L. Hanzo, "Design guidelines 733 for spatial modulation," IEEE Commun. Surveys Tuts., vol. 17, no. 1, 734 pp. 6-26, 1st Quart. 2015.

735 [21] M. Di Renzo, H. Haas, A. Ghrayeb, S. Sugiura, and L. Hanzo, "Spatial modulation for generalized MIMO: Challenges, opportunities, and implementation," Proc. IEEE, vol. 102, no. 1, pp. 56-103, Jan. 2014.

22] M. Di Renzo, H. Haas, and P. M. Grant, "Spatial modulation for multipleantenna wireless systems: A survey," IEEE Commun. Mag., vol. 49, no. 12, pp. 182-191, Dec. 2011.

23] J. Jeganathan, A. Ghrayeb, L. Szczecinski, and A. Ceron, "Space shift keying modulation for MIMO channels," IEEE Trans. Wireless Commun., vol. 8, no. 7, pp. 3692-3703, Jul. 2009.

4] M. Di Renzo and H. Haas, "Bit error probability of SM-MIMO over generalized fading channels," IEEE Trans. Veh. Technol., vol. 61, no. 3, pp. 1124-1144, Mar. 2012.

25] M. Abramowitz and I. A. Stegun, Handbook of Mathematical Functions With Formulas, Graphs, and Mathematical Tables. New York, NY, USA: Dover, 1972.

26] R. Ware and F. Lad, "Approximating the distribution for sums of products of normal variables," Univ. Canterbury, Christchurch, New Zealand, Res. Rep., 2003.

27] H. A. David and H. N. Nagaraja, Order Statistics, 3rd ed. New York, NY, USA: Wiley, 2003.

8] Evolved Universal Terrestrial Radio Access (E-UTRA); LTE Physical Layer; General Description, 3GPP TS 36.201, V11.1.0, Rel. 11, Mar. 2008.

29] X. Cong, G. Y. Li, Z. Shunqing, Y. Chen, and S. Xu, "Energy- and spectral-efficiency tradeoff in downlink OFDMA networks," IEEE Trans. Wireless Commun., vol. 10, no. 11, pp. 3874-3886, Nov. 2011.

30] A. Stavridis, S. Sinanovic, M. Di Renzo, and H. Haas, "Energy evaluation of spatial modulation at a multi-antenna base station," in Proc. IEEE 78th VTC-Fall, Sep. 2-5, 2013, pp. 1-5.

1] A. Stavridis, S. Sinanovic, M. Di Renzo, H. Haas, and P. Grant, "An energy saving base station employing spatial modulation," in Proc. IEEE 17th Int. Workshop CAMAD, Sep. 17-19, 2012, pp. 231-235.

32] S. Cui, A. J. Goldsmith, and A. Bahai, "Energy-constrained modulation optimization," IEEE Trans. Wireless Commun., vol. 4, no. 5, pp. 2349-2360, Sep. 2005.

33] C. Masouros, M. Sellathurai, and T. Ratnarajah, "Computationally efficient vector perturbation precoding using thresholded optimization," IEEE Trans. Commun., vol. 61, no. 5, pp. 1880-1890, May 2013.
[34] C. Masouros, M. Sellathurai, and T. Ratnarajah, "Maximizing energy- 773 efficiency in the vector precoded MU-MISO downlink by selective pertur- 774 bation," IEEE Trans. Wireless Commun., vol. 13, no. 9, pp. 4974-4984, 775 Sep. 2014.

[35] C. Masouros, M. Sellathurai, and T. Ratnarajah, "Vector perturbation 777 based on symbol scaling for limited feedback MISO downlinks," IEEE 778 Trans. Signal Process., vol. 62, no. 3, pp. 562-571, Feb. 1, 2014.

[36] D. Curd, "Power consumption in 65 nm FPGAs," Xilinx, San Jose, CA, 780 USA, White Paper, Feb. 2007.

[37] "W-CDMA open access small cells: Architecture, requirements and depen- 782 dencies," Small Cell Forum Ltd, Dursley, U.K., White Paper, May 2012. 783

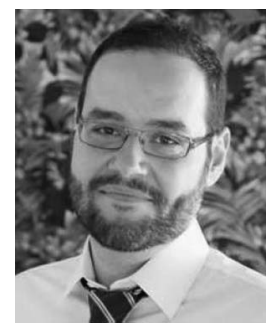

Christos Masouros (M'06-SM'14) received the 784 Diploma in electrical and computer engineering from 785 the University of Patras, Patras, Greece, in 2004 and 786 the M.Sc. degree (by research) and the Ph.D. degree 787 in electrical and electronic engineering from The 788 University of Manchester, Manchester, U.K., in 2006789 and 2009, respectively.

He is currently a Lecturer with the Department 791 of Electrical and Electronic Engineering, Univer- 792 sity College London, London, U.K. He was previ- 793 ously a Research Associate with The University of 794 Manchester and a Research Fellow with the Queen's University Belfast, Belfast, 795 U.K. His research interests lie in the field of wireless communications and 796 signal processing, with particular focus on green communications, large-scale 797 antenna systems, cognitive radio, and interference mitigation techniques for 798 multiple-input-multiple-output and multicarrier communications.

Dr. Masouros holds a Royal Academy of Engineering Research Fellowship 800 for 2011-2016 and is the Principal Investigator of the Engineering and Physical 801 Sciences Research Council's Project EP/M014150/1 on large-scale antenna sys- 802 tems. He is an Associate Editor for the IEEE COMMUNiCATIONS LETTERS. 803

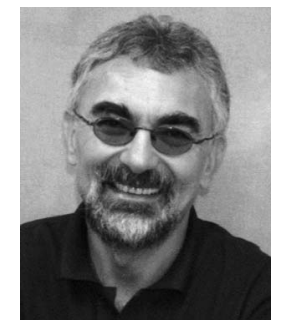

Lajos Hanzo (M'91-SM'92-F'04) received the 804 M.S. degree in electronics and the Ph.D. degree from 805 Budapest University of Technology and Economics 806 (formerly, Technical University of Budapest), 807 Budapest, Hungary, in 1976 and 1983, respectively; 808 the D.Sc. degree from the University of Southampton, 809 Southampton, U.K., in 2004; and the "Doctor Honoris 810 Causa" degree from Budapest University of Technol- 811 ogy and Economics in 2009.

During his 38-year career in telecommunications, 813 he has held various research and academic posts in 814 Hungary, Germany, and the U.K. Since 1986, he has been with the School 815 of Electronics and Computer Science, University of Southampton, where he 816 holds the Chair in Telecommunications. He is currently directing a 100-strong 817 academic research team, working on a range of research projects in the field of 818 wireless multimedia communications sponsored by industry, the Engineering 819 and Physical Sciences Research Council of U.K., the European Research 820 Merit Award. During 2008-2012, he was a Chaired Professor with Tsinghua 822 University, Beijing, China. He is an enthusiastic supporter of industrial and 823 academic liaison and offers a range of industrial courses. He has successfully 824 supervised more than $80 \mathrm{Ph} . D$. students, coauthored 20 John Wiley/IEEE Press 825 books on mobile radio communications totaling in excess of 10000 pages, and 826 published more than 1400 research entries on IEEE Xplore. He has more than 827 20000 citations. His research is funded by the European Research Council's 828 Senior Research Fellow Grant.

829

Dr. Hanzo is a Governor of the IEEE Vehicular Technology Society. He 830 has served as the Technical Program Committee Chair and the General Chair 831 of IEEE conferences, has presented keynote lectures, and has been awarded 832 a number of distinctions. During 2008-2012, he was the Editor-in-Chief of 833 the IEEE Press. He is a Fellow of the Royal Academy of Engineering, The 834 Institution of Engineering and Technology, and the European Association for 835 Signal Processing. Council's Advanced Fellow Grant, and the Royal Society Wolfson Research 821 


\section{AUTHOR QUERIES}

\section{AUTHOR PLEASE ANSWER ALL QUERIES}

AQ1 = Please check if the current affiliation of author "Christos Masouros" is properly captured, to be consistent with the biography provided; otherwise, kindly provide the correction.

AQ2 = Please check if Fig. 4 is properly cited here, to properly sequence the citations; otherwise, kindly provide the correction.

AQ3 = Please check if "a factor of" here is properly captured, to make the statement clear; otherwise, kindly provide the correction.

AQ4 = Please check if the expanded form of "FPGA" is properly captured; otherwise, kindly provide the correction.

AQ5 = Please provide publication update in Ref. [12].

\section{END OF ALL QUERIES}

